\title{
3,5,6-三取代-1,2,4-三嗪衍生物合成、表征及生物活性
}

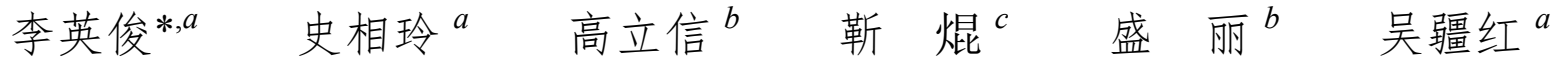 \\ 彭立娜 ${ }^{a}$ 李 佳*, $b$ \\ $\left({ }^{a}\right.$ 辽宁师范大学化学化工学院 大连 116029) \\ $\left({ }^{b}\right.$ 中国科学院上海药物研究所 国家新药笁选中心药物研究国家重点实验室 上海 201203) \\ ( ${ }^{c}$ 大连理工大学精细化工国家重点实验室 大连 116012)
}

\begin{abstract}
摘要 在 $\mathrm{NH}_{4} \mathrm{OAc} / \mathrm{HOAc}$ 存在下, 利用微波辅助, 将联苯甲酰/茴香偶酰与 2-芳氧甲基苯并咪唑-1-乙酰肼 $(3)$ 缩合, 合成 出了 30 个新的含苯并咪唑环的 3,5,6-三取代-1,2,4-三嗪衍生物 5 和 6. 利用元素分析, IR, ${ }^{1} \mathrm{H}$ NMR 及单晶 X 射线衍射进 行了结构表征. 评价了目标化合物对 Cdc25B 和 PTP1B 的抑制活性, 讨论了结构与活性的关系. 实验结果表明, 部分目 标化合物对 Cdc25B 和 PTP1B 具有良好的抑制活性, 其中 60 对 Cdc25B 的抑制活性 $\left[\mathrm{IC}_{50}=(0.84 \pm 0.22) \mu \mathrm{g} / \mathrm{mL}\right]$ 最高. 目 标化合物 $5 \mathbf{i}, 5 \mathbf{5 m}, \mathbf{5 n}, \mathbf{6 h}, \mathbf{6 j}, \mathbf{6 m}, \mathbf{6 n}$ 和 $6 \mathbf{6}$ 对 PTP1B 的抑制活性 $\left[\mathrm{IC}_{50}=(0.46 \pm 0.10) \sim(0.87 \pm 0.19) \mu \mathrm{g} / \mathrm{mL}\right]$ 均高于阳性对 照药物齐墩果酸 $\left[\mathrm{IC}_{50}=(0.97 \pm 0.15) \mu \mathrm{g} / \mathrm{mL}\right]$. 值得注意的是, 化合物 $\mathbf{5 h}, \mathbf{5 m}, \mathbf{6 n}$ 和 $\mathbf{6 o}$ 对 $\mathrm{Cdc} 25 \mathrm{~B}$ 和 PTP1B 均具有抑制 活性. 目标化合物是潜在的 Cdc25B 和 PTP1B 抑制剂.
\end{abstract}

关键词 3,5,6-三取代-1,2,4-三嗪; 苯并咪唑; 微波辐射; 合成; 晶体结构; Cdc25B 和 PTP1B 抑制剂

\section{Synthesis, Characterization and Biological Activities of 3,5,6-Trisubstituted-1,2,4-triazine Derivatives}

\author{
Li, Yingjun*,a \\ Shi, Xiangling ${ }^{a}$ \\ Gao, $\operatorname{Lixin}^{b}$ \\ Jin, $\mathrm{Kun}^{c}$ \\ Sheng, $\mathrm{Li}^{b}$ \\ $\mathrm{Wu}$, Jianghong ${ }^{a}$ \\ Peng, Lina ${ }^{a}$ \\ $\mathrm{Li}, \mathrm{Jia}^{*, b}$ \\ $\left({ }^{a}\right.$ College of Chemistry and Chemical Engineering, Liaoning Normal University, Dalian 116029) \\ $\left({ }^{b}\right.$ State Key Laboratory of Drug Research, National Center for Drug Screening, Shanghai Institute of Materia Medica, \\ Chinese Academy of Sciences, Shanghai 201203) \\ ( ${ }^{c}$ State Key Laboratory of Fine Chemicals, Dalian University of Technology, Dalian 116012)
}

\begin{abstract}
Thirty new 3,5,6-trisubstituted-1,2,4-triazine derivatives containing benzimidazole moiety (5 $\sim \mathbf{6})$ were synthesized via microwave-assisted condensation reactions of 2-aryloxymethylbenzimidazole-1-acetylhydrazines (3) with benzil $/ p$-anisil in the presence of $\mathrm{NH}_{4} \mathrm{OAc} / \mathrm{HOAc}$. The structures were characterized by elemental analysis, IR, ${ }^{1} \mathrm{H}$ NMR spectra and single crystal X-ray determination. The synthesized target compounds were evaluated for Cdc25B and PTP1B inhibitory activities. The structure-activity relationship (SAR) was also briefly discussed. The experimental results indicated that some compounds showed good inhibitory activities against Cdc25B and PTP1B. Among of them, compound $60\left[\mathrm{IC}_{50}=(0.84 \pm 0.22) \mu \mathrm{g} / \mathrm{mL}\right]$ exhibited highest inhibitory activity against $C d c 25 B$. Compounds $\mathbf{5 i}, \mathbf{5 m}, \mathbf{5 n}, \mathbf{6 h}, \mathbf{6 j}, \mathbf{6 m}, \mathbf{6 n}$ and $\mathbf{6 0}$ showed higher inhibitory activities against PTP1B $\left[\mathrm{IC}_{50}=(0.46 \pm 0.10) \sim(0.87 \pm 0.19) \mu \mathrm{g} / \mathrm{mL}\right]$ than positive control oleanolic acid $\left[\mathrm{IC}_{50}=(0.97 \pm 0.15)\right.$ $\mu \mathrm{g} / \mathrm{mL}]$. It is noteworthy that, compounds $\mathbf{5 h}, \mathbf{5 m}, \mathbf{6 n}$ and $\mathbf{6 0}$ showed inhibitory activities against Cdc25B and PTP1B. The target compounds can be considered as potential Cdc25B and PTP1B inhibitors.

Keywords 3,5,6-trisubstituted-1,2,4-triazine; benzimidazole; microwave irradiation; synthesis; crystal structure; Cdc25B and PTP1B inhibitors
\end{abstract}

1,2,4-三嗪核是重要的药效团, 在众多具有广谱生 物活性的化合物中均含有此核. 许多研究显示, 1,2,4-三
嗪衍生物具有抗癌 ${ }^{[1,2]}$ 、抗菌 ${ }^{[3]}$ 、抗真菌 ${ }^{[4]}$ 、抗炎镇痛 ${ }^{[5]}$ 、 抗 HIV-1 ${ }^{[6]}$ 、抗疮疾 ${ }^{[7]}$ 、抑制细胞分裂周期 $25 B$ 磷酸酯

\footnotetext{
* E-mail: chemlab.lnnu@163.com

Received May 21, 2014; revised revised July 25, 2014; published online September 9, 2014.
} 
酶( Cdc25B, cell division cycle 25B)和蛋白酪氨酸磷酸酯 酶 1B (PTP1B, protein tyrosine phosphatase 1B $)^{[8,9]}$ 等活 性. 某些 3,5,6-三取代-1,2,4-三嗪类化合物具有抑制血 小板聚集、抗病毒活性、抗白血病、抗卵巢癌及抗 HIV 活性 ${ }^{[10]}$. 3-取代-5,6-二芳基-1,2,4-三嗪化合物在医药和 光学材料方面具有较好的应用, 具有较好的抗癌 ${ }^{[2]}$ 、抗 炎镇痛 ${ }^{[5,11]}$ 、神经保护 ${ }^{[12,13]}$ 、抗菌 ${ }^{[14]}$ 和抗真菌活性 ${ }^{[4]}$. 作 为配体时, 可选择性地与 $\mathrm{Fe}^{3+}$ 离子络合显示出很好的荧 光效应 ${ }^{[15]}$, 与 $\mathrm{Eu}^{3+}$ 和 $\mathrm{Sm}^{3+}$ 络合后显著增强镧系元素的 光致发光性能 ${ }^{[16]}$. 在杂环化合物中, 苯并咪唑类化合物 由于其重要的生物活性以及在药物化学中的应用已成

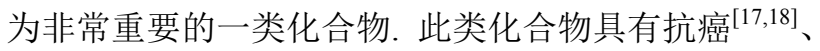

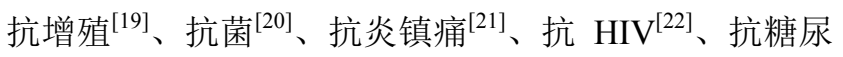
病 ${ }^{[23]}$ 和抑制 $\operatorname{Cdc} 25 \mathrm{~B}$ 和 $\mathrm{PTP} 1 \mathrm{~B}^{[8,9,24]}$ 等活性. 然而, 分子 中同时含有苯并咪唑环和 1,2,4-三嗪环的化合物文献报 道甚少. 本课题组前期的研究工作表明, 含苯并咪唑单 元的 3,6-二取代-1,2,4-三嗪衍生物具有抑制 Cdc25B 和 PTP1B 的活性 ${ }^{[8,9]}$, 是治疗癌症和糖尿病的潜在的 Cdc25B 和 PTP1B 抑制剂. 鉴于上述事实, 为了寻找高 效、低毒的新型药物, 并继续我们的研究工作, 即合成 并篎选出具较强抑制 Cdc25B 和 PTP1B 活性的小分子杂 环化合物, 本课题组在前期工作的基础上 ${ }^{[8,9]}$, 设计利用 微波辅助法合成一系列新的含苯并咪坐环的 3,5,6-三取 代-1,2,4-三嗪衍生物, 并评价目标化合物对 Cdc25B 和 PTP1B 的抑制活性, 期望目标化合物具有较好的药理活 性和应用前景. 目标化合物的合成路线见 Scheme 1.

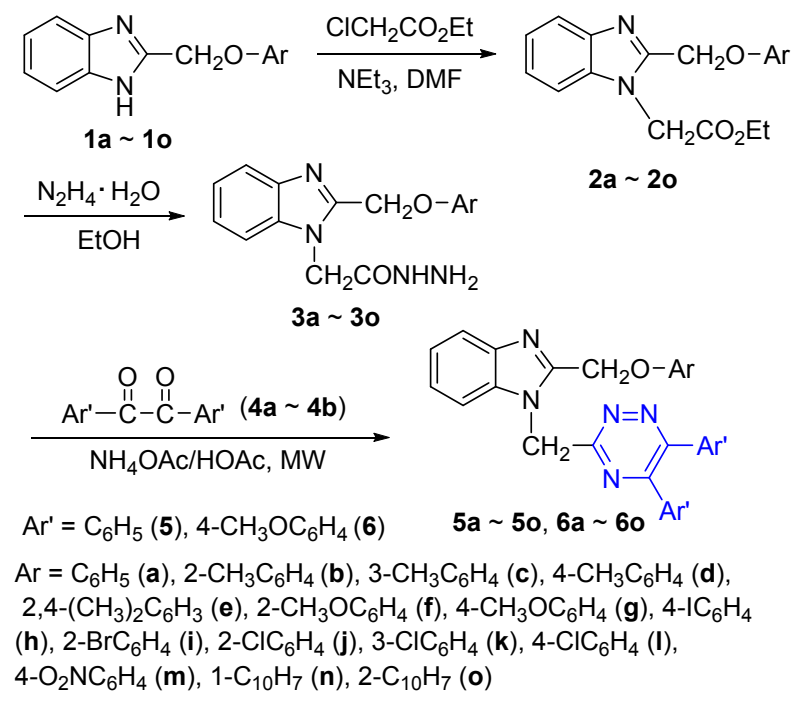

图式 1 化合物 5a $\sim 50$ 和 $6 \mathrm{a} \sim 60$ 的合成路线

Scheme 1 Synthetic route of compounds $5 a \sim 50$ and $\mathbf{6 a} \sim \mathbf{6 0}$

\section{1 结果与讨论}

\section{1 目标化合物 5 和 6 的合成}

参考文献[25], 采用微波辐射技术, 在冰乙酸介质
中，中间体化合物 3 与联苯甲酰/茴香偶酰、醋酸铵缩合 反应，成功地合成出了目标化合物 5 和 $\mathbf{6}$. 与经典方法 相比较, 微波法具有反应时间短、产率较高等优点(见表 1). 另外, 我们在相同的条件(即在 $100{ }^{\circ} \mathrm{C}$, 反应 40 $\mathrm{min}$ )、而无微波辐射作用下，进行了此反应，结果没有 得到 1,2,4-三嗪产物, 这说明了微波条件对此反应的必 要性.

\section{2 目标化合物 5 和 6 的波谱特征}

目标化合物的 IR 数据显示, 化合物 5 和 $\mathbf{6}$ 在 3010 $3082 \mathrm{~cm}^{-1}$ 处出现苯环不饱和 $\mathrm{C}-\mathrm{H}$ 伸缩振动吸收峰, 在 $2925 \sim 2961$ 和 $2834 \sim 2870 \mathrm{~cm}^{-1}$ 处出现饱和 $\mathrm{C}-\mathrm{H}$ 伸缩 振动吸收峰, 在 $1605 \sim 1631 \mathrm{~cm}^{-1}$ 处出现 $\mathrm{C}=\mathrm{N}$ 伸缩振 动吸收峰, 在 $1455 \sim 1602 \mathrm{~cm}^{-1}$ 处出现苯环骨架伸缩振 动吸收峰, 在 $1219 \sim 1268$ 和 $1010 \sim 1154 \mathrm{~cm}^{-1}$ 处出现 $\mathrm{C}-\mathrm{O}$ 伸缩振动吸收峰, 在 $1510 \sim 1517$ 和 $1340 \sim 1344$ $\mathrm{cm}^{-1}$ 处出现 $\mathrm{NO}_{2}$ 伸缩振动吸收峰. 未见原料酰肼 $\mathrm{N}-\mathrm{H}$ 及邻二酮和酰肼的 $\mathrm{C}=\mathrm{O}$ 伸缩振动吸收峰.

目标化合物的 ${ }^{1} \mathrm{H}$ NMR 数据显示, 在 $\delta \quad 6.60 \sim 8.05$ 处出现的吸收峰为苯并咪唑环和芳环的质子吸收峰, 在 $\delta 6.04 \sim 6.22$ 处出现的吸收峰为 $\mathrm{NCH}_{2}$ 的质子吸收峰, 在 $\delta 5.45 \sim 5.76$ 处出现的吸收峰为 $\mathrm{CH}_{2} \mathrm{O}$ 的质子吸收峰, 在 $\delta 3.56 \sim 3.79$ 处出现的吸收峰为 $\mathrm{CH}_{3} \mathrm{O}$ 的质子吸收峰, 在 $\delta 1.73 \sim 2.25$ 处出现的吸收峰为 $\mathrm{CH}_{3}$ 的质子吸收峰.

上述数据分析结果与化合物结构一致, 这表明已成 功合成了目标化合物.

\section{3 目标化合物 $5 \mathrm{~g}$ 晶体的物化数据、晶体结构解析及 结果}

在 DMF-EtOH 中, 采用化合物溶液缓慢蒸发的方 法, 生长 $5 \mathrm{~g}$ 的结晶. 选取合适尺寸的荧光黄色目标化合 物 5g 的晶体放置于 Bruker AXS SMART APEX II CCD 型 $\mathrm{X}$ 射线单晶衍射仪上进行测试, 测试温度为 $23{ }^{\circ} \mathrm{C}$. 利用经石墨单色化的 Mo K $\alpha$ 射线 $(\lambda=0.71073 \AA$ )进行衍 射数据收集. 采用直接法和差值 Fourier 合成法解出晶 体结构. 采用全矩阵, 最小二乘法修正. 氢原子坐标由 理论加氢程序获得. 全部结构计算均用 SHELX 97 程 序测定. 测得结构如图 1 所示, 由此进一步确定了目标 化合物结构的正确性.

单晶 $X$ 射线衍射实验测定得该化合物的分子式为: $\mathrm{C}_{31} \mathrm{H}_{25} \mathrm{~N}_{5} \mathrm{O}_{2}, M=499.56$, 为正交晶系, 属于 $P b c a$ 空间 群, 晶胞参数为 $a=17.669(2) \AA, \alpha=90^{\circ}, b=9.0704(11)$ $\AA, \beta=90^{\circ}, c=31.672(4) \AA, \gamma=90^{\circ}, V=5075.8(11) \AA^{3}$, $D_{\mathrm{c}}=1.307 \mathrm{Mg} / \mathrm{m}^{3}$, 晶胞中分子数: $Z=8, F(000)=2096$, 吸收系数 $\mu=0.084 \mathrm{~mm}^{-1}, R_{1}=0.0423[I>2 \sigma(\mathrm{I})], w R_{2}=$ $0.1155, s=1.048$. 数据存于英国剑桥数据中心, $\mathrm{CCDC}$ 号为 999805 . 


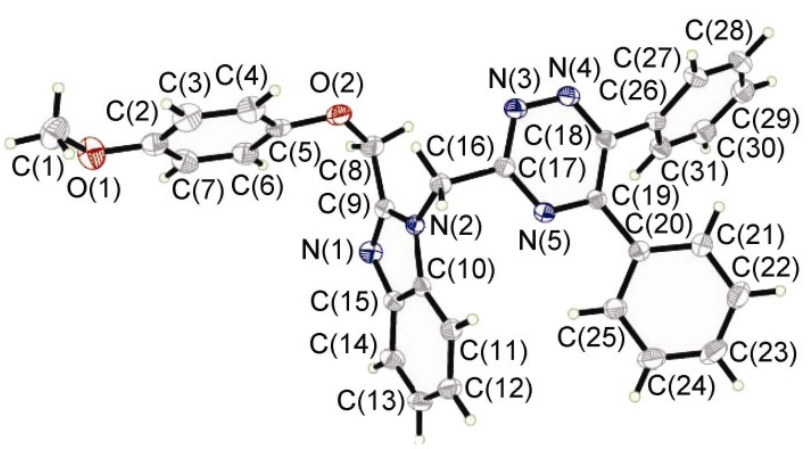

图 1 化合物 $\mathbf{5 g}$ 的晶体结构(ORTEP 图)

Figure 1 Crystal stractre (ORTEP diagram) of compound $\mathbf{5 g}$

由单晶数据可知, 三嗪环 5-位和 6-位上两个苯环不 在同一平面内, 两者之间的夹角是 $63.2^{\circ}$, 并且二者与三 嗪环均存在着一定的二面角, 分别为 $23.7^{\circ}$ 和 $29.1^{\circ}$. 此 外, $\mathrm{C}(17)-\mathrm{C}(16)-\mathrm{N}(2)-\mathrm{C}(10)$ 的扭转角为 $84.9(12)^{\circ}$,
$\mathrm{N}(1)-\mathrm{C}(9)-\mathrm{C}(8)-\mathrm{O}(2)$ 的扭转角为 $125.0(11)^{\circ}$, 由此确 定了苯并咪坐环 1-位和 2-位上两个取代基的方向.

\section{4 生物活性}

对所合成的新目标化合物 $5 a \sim 50$ 和 $6 \mathbf{6} \sim \mathbf{6 0}$ 进行了 Cdc25B 和 PTP1B 抑制活性的笁选, 对照药物分别为正 钒酸钠和齐墩果酸. 初笁选择单浓度 $(5 \mu \mathrm{g} / \mathrm{mL})$ 条件下, 对样品的活性进行测试. 对于抑制率 $>50 \%$ 的样品, 再 测试活性剂量依赖关系, 即 $\mathrm{IC}_{50}$ 值, 其测试结果见表 1 .

由测试结果可以看出，目标化合物 $5 \mathrm{~h} 、 5 \mathrm{~m} 、 6 \mathbf{f} 、 6 \mathrm{n}$ 和 60 对 $\mathrm{Cdc} 25 \mathrm{~B}$ 表现出良好的抑制活性 $\left[\mathrm{IC}_{50}=(0.84 \pm\right.$ $0.22) \sim(3.54 \pm 0.61) \mu \mathrm{g} / \mathrm{mL}$ ], 其中化合物 60 对 Cdc $25 B$ 的抑制活性最高，但其抑制活性均低于对照药物正钒酸 钠 $\left[\mathrm{IC}_{50}=(0.28 \pm 0.03) \mu \mathrm{g} / \mathrm{mL}\right]$.

目标化合物 $5 \mathrm{~h} \sim 5 \mathbf{j}, 5 \mathrm{5l} \sim \mathbf{5 n}, \mathbf{6 e}, 6 \mathrm{~h}, \mathbf{6 j}, 6 \mathrm{ll} \sim 60$ 对 PTP1B 表现出良好的抑制活性 $\left[\mathrm{IC}_{50}=(0.46 \pm 0.10) \sim\right.$

表 1 微波辐射下 3,5,6-三取代-1,2,4-三嗪衍生物的产率及对 Cdc25B 和 PTP1B 的抑制活性 ${ }^{a}$

Table 1 Yields of 3,5,6-trisubstituted-1,2,4-triazines under microwave radiation and inhibition activities against Cdc25B and PTP1B

\begin{tabular}{|c|c|c|c|c|c|c|c|}
\hline \multirow{2}{*}{ Compd. } & \multirow{2}{*}{$\mathrm{Ar}^{\prime}$} & \multirow{2}{*}{$\mathrm{Ar}$} & \multirow{2}{*}{ Yield $^{b} / \%$} & \multicolumn{2}{|c|}{$\mathrm{Cdc} 25 \mathrm{~B}$} & \multicolumn{2}{|c|}{ PTP1B } \\
\hline & & & & Inhibition $^{a} / \%$ & $\mathrm{IC}_{50}\left(\mu \mathrm{g} \cdot \mathrm{mL}^{-1}\right)$ & Inhibition $^{a} / \%$ & $\mathrm{IC}_{50} /\left(\mu \mathrm{g} \cdot \mathrm{mL}^{-1}\right)$ \\
\hline $5 a$ & $\mathrm{C}_{6} \mathrm{H}_{5}$ & $\mathrm{C}_{6} \mathrm{H}_{5}$ & 76 & $40.14 \pm 8.28$ & $\mathrm{NA}^{c}$ & $6.07 \pm 2.25$ & NA \\
\hline $5 b$ & $\mathrm{C}_{6} \mathrm{H}_{5}$ & $2-\mathrm{CH}_{3} \mathrm{C}_{6} \mathrm{H}_{4}$ & 80 & $47.34 \pm 4.08$ & NA & $16.15 \pm 10.34$ & NA \\
\hline $5 c$ & $\mathrm{C}_{6} \mathrm{H}_{5}$ & $3-\mathrm{CH}_{3} \mathrm{C}_{6} \mathrm{H}_{4}$ & 77 & $36.13 \pm 10.79$ & NA & $45.19 \pm 7.00$ & NA \\
\hline $5 d$ & $\mathrm{C}_{6} \mathrm{H}_{5}$ & 4- $\mathrm{CH}_{3} \mathrm{C}_{6} \mathrm{H}_{4}$ & 83 & $33.21 \pm 6.77$ & NA & $42.89 \pm 5.53$ & NA \\
\hline $5 e$ & $\mathrm{C}_{6} \mathrm{H}_{5}$ & $2,4-\left(\mathrm{CH}_{3}\right)_{2} \mathrm{C}_{6} \mathrm{H}_{4}$ & 73 & $46.53 \pm 6.50$ & NA & $23.97 \pm 11.77$ & NA \\
\hline $5 f$ & $\mathrm{C}_{6} \mathrm{H}_{5}$ & $2-\mathrm{CH}_{3} \mathrm{OC}_{6} \mathrm{H}_{4}$ & 72 & $25.74 \pm 5.50$ & NA & $23.11 \pm 3.25$ & NA \\
\hline $5 \mathrm{~g}$ & $\mathrm{C}_{6} \mathrm{H}_{5}$ & 4- $\mathrm{CH}_{3} \mathrm{OC}_{6} \mathrm{H}_{4}$ & 79 & $46.99 \pm 0.91$ & NA & $19.07 \pm 13.83$ & NA \\
\hline $5 \mathrm{~h}$ & $\mathrm{C}_{6} \mathrm{H}_{5}$ & $4-\mathrm{IC}_{6} \mathrm{H}_{4}$ & 70 & $99.30 \pm 7.73$ & $3.54 \pm 0.61$ & $79.34 \pm 3.42$ & $1.52 \pm 0.28$ \\
\hline $5 \mathbf{i}$ & $\mathrm{C}_{6} \mathrm{H}_{5}$ & $2-\mathrm{BrC}_{6} \mathrm{H}_{4}$ & 73 & $37.03 \pm 2.33$ & NA & $89.63 \pm 10.54$ & $0.80 \pm 0.19$ \\
\hline $5 \mathbf{j}$ & $\mathrm{C}_{6} \mathrm{H}_{5}$ & $2-\mathrm{ClC}_{6} \mathrm{H}_{4}$ & $77(49)^{d}$ & $14.73 \pm 1.35$ & NA & $95.95 \pm 0.83$ & $1.54 \pm 0.21$ \\
\hline $5 \mathbf{k}$ & $\mathrm{C}_{6} \mathrm{H}_{5}$ & $3-\mathrm{ClC}_{6} \mathrm{H}_{4}$ & $76(39)^{d}$ & $75.41 \pm 5.33$ & NA & $24.44 \pm 6.45$ & NA \\
\hline $5 \mathbf{l}$ & $\mathrm{C}_{6} \mathrm{H}_{5}$ & $4-\mathrm{ClC}_{6} \mathrm{H}_{4}$ & $78(47)^{d}$ & $69.50 \pm 9.85$ & NA & $61.10 \pm 12.69$ & $1.54 \pm 0.31$ \\
\hline $5 \mathrm{~m}$ & $\mathrm{C}_{6} \mathrm{H}_{5}$ & $4-\mathrm{O}_{2} \mathrm{NC}_{6} \mathrm{H}_{4}$ & 70 & $93.41 \pm 3.08$ & $3.27 \pm 0.43$ & $85.01 \pm 2.70$ & $0.76 \pm 0.27$ \\
\hline $5 n$ & $\mathrm{C}_{6} \mathrm{H}_{5}$ & $1-\mathrm{C}_{10} \mathrm{H}_{7}$ & 74 & $12.83 \pm 5.54$ & NA & $65.76 \pm 3.49$ & $0.73 \pm 0.18$ \\
\hline 50 & $\mathrm{C}_{6} \mathrm{H}_{5}$ & $2-\mathrm{C}_{10} \mathrm{H}_{7}$ & 75 & $41.83 \pm 1.52$ & NA & $19.67 \pm 15.22$ & NA \\
\hline $6 a$ & 4- $\mathrm{MeOC}_{6} \mathrm{H}_{4}$ & $\mathrm{C}_{6} \mathrm{H}_{5}$ & 72 & $27.09 \pm 3.38$ & NA & $32.67 \pm 5.16$ & NA \\
\hline $6 b$ & 4- $\mathrm{MeOC}_{6} \mathrm{H}_{4}$ & $2-\mathrm{CH}_{3} \mathrm{C}_{6} \mathrm{H}_{4}$ & 73 & $20.46 \pm 5.59$ & NA & $18.60 \pm 9.83$ & NA \\
\hline $6 c$ & 4- $\mathrm{MeOC}_{6} \mathrm{H}_{4}$ & $3-\mathrm{CH}_{3} \mathrm{C}_{6} \mathrm{H}_{4}$ & 70 & $46.79 \pm 1.16$ & NA & $40.20 \pm 8.22$ & NA \\
\hline 6d & $4-\mathrm{MeOC}_{6} \mathrm{H}_{4}$ & $4-\mathrm{CH}_{3} \mathrm{C}_{6} \mathrm{H}_{4}$ & 81 & $22.56 \pm 2.22$ & NA & $47.18 \pm 10.02$ & NA \\
\hline $6 e$ & 4- $\mathrm{MeOC}_{6} \mathrm{H}_{4}$ & $2,4-\left(\mathrm{CH}_{3}\right)_{2} \mathrm{C}_{6} \mathrm{H}_{4}$ & 71 & $39.83 \pm 4.04$ & NA & $70.74 \pm 8.63$ & $1.27 \pm 0.24$ \\
\hline $6 f$ & 4- $\mathrm{MeOC}_{6} \mathrm{H}_{4}$ & $2-\mathrm{CH}_{3} \mathrm{OC}_{6} \mathrm{H}_{4}$ & 71 & $97.73 \pm 1.93$ & $2.51 \pm 0.12$ & $30.88 \pm 3.16$ & NA \\
\hline $6 g$ & $4-\mathrm{MeOC}_{6} \mathrm{H}_{4}$ & $4-\mathrm{CH}_{3} \mathrm{OC}_{6} \mathrm{H}_{4}$ & 76 & $24.51 \pm 3.34$ & NA & $46.73 \pm 2.31$ & NA \\
\hline $6 \mathrm{~h}$ & 4- $\mathrm{MeOC}_{6} \mathrm{H}_{4}$ & $4-\mathrm{IC}_{6} \mathrm{H}_{4}$ & 70 & $29.43 \pm 10.98$ & NA & $70.46 \pm 12.44$ & $0.82 \pm 0.22$ \\
\hline $6 \mathbf{i}$ & 4- $\mathrm{MeOC}_{6} \mathrm{H}_{4}$ & $2-\mathrm{BrC}_{6} \mathrm{H}_{4}$ & 69 & $35.19 \pm 1.74$ & NA & $42.77 \pm 14.13$ & NA \\
\hline $6 \mathbf{j}$ & $4-\mathrm{MeOC}_{6} \mathrm{H}_{4}$ & $2-\mathrm{ClC}_{6} \mathrm{H}_{4}$ & 71 & $31.15 \pm 2.73$ & NA & $82.72 \pm 5.08$ & $0.87 \pm 0.19$ \\
\hline $6 \mathbf{k}$ & $4-\mathrm{MeOC}_{6} \mathrm{H}_{4}$ & $3-\mathrm{ClC}_{6} \mathrm{H}_{4}$ & 70 & $18.98 \pm 3.96$ & NA & $41.33 \pm 11.13$ & NA \\
\hline 61 & $4-\mathrm{MeOC}_{6} \mathrm{H}_{4}$ & $4-\mathrm{ClC}_{6} \mathrm{H}_{4}$ & 69 & $43.55 \pm 2.60$ & NA & $57.65 \pm 4.47$ & $1.74 \pm 0.85$ \\
\hline $6 m$ & $4-\mathrm{MeOC}_{6} \mathrm{H}_{4}$ & $4-\mathrm{O}_{2} \mathrm{NC}_{6} \mathrm{H}_{4}$ & 68 & $43.47 \pm 2.77$ & NA & $83.47 \pm 3.26$ & $0.60 \pm 0.12$ \\
\hline $6 n$ & 4- $\mathrm{MeOC}_{6} \mathrm{H}_{4}$ & $1-\mathrm{C}_{10} \mathrm{H}_{7}$ & 70 & $96.50 \pm 0.26$ & $1.71 \pm 0.60$ & $75.34 \pm 1.18$ & $0.46 \pm 0.10$ \\
\hline 60 & 4- $\mathrm{MeOC}_{6} \mathrm{H}_{4}$ & $2-\mathrm{C}_{10} \mathrm{H}_{7}$ & 72 & $98.51 \pm 0.54$ & $0.84 \pm 0.22$ & $86.94 \pm 2.57$ & $0.62 \pm 0.23$ \\
\hline Ref. & & & & & $0.28 \pm 0.03^{e}$ & & $0.97 \pm 0.15^{f}$ \\
\hline
\end{tabular}

${ }^{a}$ 目标化合物的测试浓度为 $5 \mu \mathrm{g} / \mathrm{mL}$, 均溶于 DMSO 中; ${ }^{b}$ 分离纯化后的产率; ${ }^{c} \mathrm{NA}$ 表示没有活性; ${ }^{d}$ 括号内的数据为采用经典法合成所得 ${ }^{[25]}$, 反应条件: 在 $\mathrm{NH}_{4} \mathrm{OAc} / \mathrm{HOAc}$ 存在下, 联苯甲酰(4a) 与取代的酰胖(3)缩合, 回流反应 $20 \mathrm{~h} ;{ }^{e}$ 阳性对照物是 $\mathrm{Na}_{3} \mathrm{VO}_{4} ;{ }^{f}$ 阳性对照物是齐墩果酸. 
$(1.74 \pm 0.85) \mu \mathrm{g} / \mathrm{mL}$ ], 其中, 化合物 6n 对 PTP1B 显示出 最高的抑制活性 $\left[\mathrm{IC}_{50}=(0.46 \pm 0.10) \mu \mathrm{g} / \mathrm{mL}\right]$. 在活性化 合物中, 5i, 5m, 5n, 6h, 6j, 6m, 6n 和 60 对 PTP1B 的抑制 活性 $\left[\mathrm{IC}_{50}=(0.46 \pm 0.10) \sim(0.87 \pm 0.19) \mu \mathrm{g} / \mathrm{mL}\right]$ 均高于阳 性对照药物齐墩果酸 $\left[\mathrm{IC}_{50}=(0.97 \pm 0.15) \mu \mathrm{g} / \mathrm{mL}\right]$. 值得 注意的是, 化合物 $5 \mathbf{h}, \mathbf{5 m}, \mathbf{6 n}$ 和 60 对 Cdc25B 和 PTP1B 均具有抑制活性.

对表 1 中抑制率数据的研究可知结构与活性的关 系. 化合物 5 和 6 对 $\mathrm{Cdc} 25 \mathrm{~B}$ 的抑制活性与 $\mathrm{Ar}$ 的结构以 及 $A r^{\prime}$ 中苯环的修饰有关系. 当 $A r=$ 稠环时, $A r^{\prime}$ 中苯环 的 4-位引入 $\mathrm{CH}_{3} \mathrm{O}$ 时活性大大增强, 且高于 $\mathrm{Ar}^{\prime}$ 中苯环上 无取代基的对应化合物的活性 (6n 和 $\mathbf{6 0}$ 的抑制率高于 $5 \mathbf{n}$ 和 5o); 当 $\mathrm{Ar}=$ (未)取代的苯环时(即非稠环), $A r^{\prime}$ 中苯 环上 4-位引入 $\mathrm{CH}_{3} \mathrm{O}$ 后(即 $\mathrm{Ar}=4-\mathrm{CH}_{3} \mathrm{OC}_{6} \mathrm{H}_{4}$ ), 化合物 6 的活性普遍低于相对应 $\mathrm{Ar}^{\prime}=\mathrm{C}_{6} \mathrm{H}_{5}$ 的化合物 5 的活性 $\left(\mathrm{Ar}=2-\mathrm{CH}_{3} \mathrm{OC}_{6} \mathrm{H}_{4}\right.$ 除外, 此时 $6 \mathbf{f}$ 的活性高于 $\left.\mathbf{5 f}\right)$. 当 $\mathrm{Ar}^{\prime}=\mathrm{C}_{6} \mathrm{H}_{5} / 4-\mathrm{CH}_{3} \mathrm{OC}_{6} \mathrm{H}_{4}$ 时, $\mathrm{Ar}=$ (未)取代的苯环时(即非 稠环), 且 $\mathrm{Ar}$ 的苯环 4-位上引入吸电子基团时活性较高 $(5 \mathrm{~h}$ 和 $5 \mathrm{~m}$ 的活性高于 $5 \mathrm{~d}$ 和 $5 \mathrm{~g}, 6 \mathrm{~d}$ 和 $6 \mathrm{~m}$ 的活性高于 $6 \mathrm{~d}$ 和 $6 \mathrm{~g}$ ).

同样，化合物 5 和 6 对 PTP1B 的抑制活性与 Ar 的 结构以及 $\mathrm{Ar}^{\prime}$ 中苯环的修饰有关系. 当 $\mathrm{Ar}^{\prime}=$ $\mathrm{C}_{6} \mathrm{H}_{5} / 4-\mathrm{CH}_{3} \mathrm{OC}_{6} \mathrm{H}_{4}$ 时, $\mathrm{Ar}=$ 稠环或吸电子基团取代的苯 环时 $\left(\mathrm{Ar}=3-\mathrm{ClC}_{6} \mathrm{H}_{4}\right.$ 除外), 抑制率较高. 当 $\mathrm{Ar}^{\prime}=4-$ $\mathrm{CH}_{3} \mathrm{OC}_{6} \mathrm{H}_{4}$ 时, 化合物 6 的抑制活性高于相对应的 $\mathrm{Ar}^{\prime}=$ $\mathrm{C}_{6} \mathrm{H}_{5}$ 化合物 5 的抑制活性.

\section{2 结论}

采用微波辐射辅助, 在 $\mathrm{NH}_{4} \mathrm{OAc} / \mathrm{HOAc}$ 存在下, 将 酰肼和联苯甲酰/茴香偶酰缩合, 合成出了 30 个新的含 苯并咪唑单元的 3,5,6-三取代-1,2,4-三嗪衍生物 $5 \sim 6$. 与经典方法相比较, 该合成方法具有反应时间短、操作 简便、产率较高等优点. 对所合成的目标化合物进行了 Cdc25B 和 PTP1B 抑制活性的篮选. 实验结果表明, 部 分目标化合物对 Cdc25B 和 PTP1B 表现出良好的抑制活 性, 并且部分目标化合物对 PTP1B 的抑制活性高于对 照药物. 目标化合物可作为治疗癌症和糖尿病的潜在的 Cdc25B 和 PTP1B 抑制剂.

\section{3 实验部分}

\section{1 仪器与试剂}

X-5 显微熔点测定仪 (温度计未经校正); 德国 TENSOR 27 型 FT-IR 红外光谱仪, KBr 压片; 美国 Varian INOVA $400 \mathrm{MHz}$ 核磁共振仪, 德国 BRUKER $500 \mathrm{MHz}$ 核磁共振仪, DMSO- $d_{6}$ 为溶剂, TMS 为内标;
德国 Elementar 公司 Vario EL 型元素分析仪; 德国 Bruker Smart APEX II 型单晶 X 射线衍射仪; 北京祥鹄 科技发展有限公司 XH-100B 型电脑微波催化合成/萃 取仪. 所用试剂均为分析纯.

\section{2 合成}

\subsection{1 中间体的合成}

2-芳氧甲基苯并咪唑(1)、2-芳氧甲基苯并咪唑-1乙酸乙酯(2)和 2-芳氧甲基苯并咪唑-1-乙酰肼(3)按照参 考文献[24]合成.

\subsection{2目标化合物 $5 \sim 6$ 的合成}

在干燥的 $50 \mathrm{~mL}$ 三口瓶中加入 $1 \mathrm{mmol}$ 联苯甲酰/ 茴香偶酰 $(\mathbf{4 a} / \mathbf{4 b}) 、 1 \mathrm{mmol}$ 酰肼(3)、26 $\mathrm{mmol}$ 乙酸铵和 2 $\mathrm{mL}$ 冰醋酸, 在 $500 \mathrm{~W}, 100{ }^{\circ} \mathrm{C}$ 条件下, 微波辐射反应 $40 \mathrm{~min}$. 然后, 将冷却至室温的反应混合物倾入含有 $100 \mathrm{~mL}$ 水的烧杯中, 摚拌下用氨水调节溶液 $\mathrm{pH}=8 \sim 9$, 抽滤, 固体用水洗至中性, 干燥后得到黄色粉末状固体 粗产品. 粗品用柱层析法分离提纯 $[V$ (乙酸乙酯)： $V$ (石 油醚 $)=1: 5$ ]或用乙醇重结晶后即得到纯品目标化合 物.

3-[2-(苯氧甲基)苯并咪唑-1-亚甲基]-5,6-二苯基1,2,4-三嗪(5a): 荧光黄色晶体, 收率 76\%. m.p. 123.8 $124.6{ }^{\circ} \mathrm{C} ;{ }^{1} \mathrm{H}$ NMR (400 MHz, DMSO- $\left.d_{6}\right) \delta: 5.63(\mathrm{~s}, 2 \mathrm{H}$, $\left.\mathrm{CH}_{2} \mathrm{O}\right), 6.22\left(\mathrm{~s}, 2 \mathrm{H}, \mathrm{NCH}_{2}\right), 6.94(\mathrm{~d}, J=8.4 \mathrm{~Hz}, 2 \mathrm{H}$, Ar-H), 7.00 (t, $J=7.6 \mathrm{~Hz}, 1 \mathrm{H}, \mathrm{Ar}-\mathrm{H}), 7.22 \sim 7.44(\mathrm{~m}, 8 \mathrm{H}$, BZM- $\left.\mathrm{H}_{5}, \mathrm{H}_{6}, \quad \mathrm{Ar}-\mathrm{H} \times 6\right), 7.44 \sim 7.60(\mathrm{~m}, 6 \mathrm{H}, \quad \mathrm{Ar}-\mathrm{H} \times 2$, Ar'-H×4), $7.82\left(\mathrm{~d}, J=7.2 \mathrm{~Hz}, 2 \mathrm{H}, \mathrm{BZM}-\mathrm{H}_{4}, \mathrm{H}_{7}\right)$; IR $(\mathrm{KBr})$ v: 3058, 3032, 2938, 2853, 1618 (W), 1598, 1494, 1461, $1239,1032 \mathrm{~cm}^{-1}$. Anal. calcd for $\mathrm{C}_{30} \mathrm{H}_{23} \mathrm{~N}_{5} \mathrm{O}: \mathrm{C} 76.74, \mathrm{H}$ 4.94, N 14.92; found C 76.97, H 5.09, N 14.73.

3-[2-(2-甲基苯氧甲基)苯并咪唑-1-亚甲基]-5,6-二 苯基-1,2,4-三嗪(5b): 黄色晶体, 收率 80\%. m.p. 149.7 $150.7{ }^{\circ} \mathrm{C} ;{ }^{1} \mathrm{H}$ NMR (400 MHz, DMSO- $\left.d_{6}\right) \delta: 1.76$ (s, 3H, $\left.\mathrm{CH}_{3}\right), 5.55\left(\mathrm{~s}, 2 \mathrm{H}, \mathrm{CH}_{2} \mathrm{O}\right), 6.16\left(\mathrm{~s}, 2 \mathrm{H}, \mathrm{NCH}_{2}\right), 6.80$ (t, $J=$ $7.4 \mathrm{~Hz}, 1 \mathrm{H}, \mathrm{Ar}-\mathrm{H}), 7.02$ (d, $J=7.6 \mathrm{~Hz}, 1 \mathrm{H}, \mathrm{Ar}-\mathrm{H}), 7.11$ (t, $J=7.6 \mathrm{~Hz}, 1 \mathrm{H}, \quad$ Ar-H), $7.15 \sim 7.22(\mathrm{~m}, 3 \mathrm{H}, \quad$ Ar- $\mathrm{H}$, Ar'-H $\times 2), 7.22 \sim 7.32\left(\mathrm{~m}, 4 \mathrm{H}, \mathrm{BZM}-\mathrm{H}_{5}, \mathrm{H}_{6}, \mathrm{Ar}-\mathrm{H} \times 2\right)$, $7.34 \sim 7.48\left(\mathrm{~m}, 6 \mathrm{H}, \operatorname{Ar}^{\prime}-\mathrm{H}\right), 7.69(\mathrm{~d}, J=7.6 \mathrm{~Hz}, 1 \mathrm{H}$, BZM-H $), 7.73\left(\right.$ d, $\left.J=7.2 \mathrm{~Hz}, 1 \mathrm{H}, \mathrm{BZM}-\mathrm{H}_{4}\right)$; IR $(\mathrm{KBr}) v$ : 3059, 3030, 2948, 2857, 1618 (W), 1602, 1494, 1464, $1239,1052 \mathrm{~cm}^{-1}$. Anal. calcd for $\mathrm{C}_{31} \mathrm{H}_{25} \mathrm{~N}_{5} \mathrm{O}: \mathrm{C} 77.00, \mathrm{H}$ 5.21, N 14.48; found C 76.82, H 5.33, N 14.26.

3-[2-(3-甲基苯氧甲基)苯并咪唑-1-亚甲基]-5,6-二 苯基-1,2,4-三嗪(5c): 苂光黄色晶体, 收率 77\%. m.p. $100.7 \sim 101.7{ }^{\circ} \mathrm{C} ;{ }^{1} \mathrm{H}$ NMR (400 MHz, DMSO- $d_{6}$ ) $\delta: 2.14$ (s, $\left.3 \mathrm{H}, \mathrm{CH}_{3}\right), 5.49$ (s, $\left.2 \mathrm{H}, \mathrm{CH}_{2} \mathrm{O}\right), 6.12\left(\mathrm{~s}, 2 \mathrm{H}, \mathrm{NCH}_{2}\right), 6.60$ 
(s, 1H, Ar-H), 6.65 (d, J=8.0 Hz, 1H, Ar-H), 6.70 (d, $J=$ $7.2 \mathrm{~Hz}, 1 \mathrm{H}, \mathrm{Ar}-\mathrm{H}), 7.07$ (t, $J=8.0 \mathrm{~Hz}, 1 \mathrm{H}, \mathrm{Ar}-\mathrm{H}), 7.22 \sim$ $7.34\left(\mathrm{~m}, 6 \mathrm{H}, \mathrm{BZM}-\mathrm{H}_{5}, \mathrm{H}_{6}, \mathrm{Ar}-\mathrm{H} \times 4\right), 7.38 \sim 7.50(\mathrm{~m}, 6 \mathrm{H}$, Ar'-H), $7.68 \sim 7.76\left(\mathrm{~m}, 2 \mathrm{H}, \mathrm{BZM}-\mathrm{H}_{4}, \mathrm{H}_{7}\right)$; IR (KBr) $v$ : 3059, 3030, 2948, 2859, 1615 (W), 1602, 1582, 1494, 1461, 1259, $1154 \mathrm{~cm}^{-1}$. Anal. calcd for $\mathrm{C}_{31} \mathrm{H}_{25} \mathrm{~N}_{5} \mathrm{O}: \mathrm{C}$ 77.00, H 5.21, N 14.48; found C 77.21, H 5.04, N 14.34.

3-[2-(4-甲基苯氧甲基)苯并咪唑-1-亚甲基]-5,6-二 苯基-1,2,4-三嗪(5d): 苂光黄色晶体, 收率 $83 \%$. m.p. $146.0 \sim 147.5{ }^{\circ} \mathrm{C} ;{ }^{1} \mathrm{H}$ NMR (400 MHz, DMSO- $\left.d_{6}\right) \delta: 2.25$ (s, 3H, $\left.\mathrm{CH}_{3}\right), 5.59$ (s, 2H, $\left.\mathrm{CH}_{2} \mathrm{O}\right), 6.21$ (s, 2H, $\left.\mathrm{NCH}_{2}\right), 6.82$ (d, $J=8.0 \mathrm{~Hz}, 2 \mathrm{H}, \mathrm{Ar}-\mathrm{H}), 7.07$ (d, $J=8.0 \mathrm{~Hz}, 2 \mathrm{H}, \mathrm{Ar}-\mathrm{H})$, $7.30 \sim 7.44\left(\mathrm{~m}, 6 \mathrm{H}, \mathrm{BZM}-\mathrm{H}_{5}, \mathrm{H}_{6}, \mathrm{Ar}-\mathrm{H} \times 4\right), 7.48 \sim 7.60$ (m, 6H, Ar'-H), 7.82 (d, J=8.0 Hz, 2H, BZM-H ${ }_{4} \mathrm{H}_{7}$ ); IR (KBr) v: 3059, 3030, 2948, 2859, 1615 (W), 1588, 1510, 1461, 1236, $1030 \mathrm{~cm}^{-1}$. Anal. calcd for $\mathrm{C}_{31} \mathrm{H}_{25} \mathrm{~N}_{5} \mathrm{O}: \mathrm{C}$ 77.00, H 5.21, N 14.48; found C 77.16, H 5.01, N 14.59.

3-[2-(2,4-二甲基苯氧甲基)苯并咪唑-1-亚甲基]-5,6二苯基-1,2,4-三嗪(5e): 黄色晶体, 收率 73\%. m.p. $143.8 \sim 144.3{ }^{\circ} \mathrm{C} ;{ }^{1} \mathrm{H}$ NMR (400 MHz, DMSO- $\left.d_{6}\right) \delta: 1.74$ $\left(\mathrm{s}, 3 \mathrm{H}, \mathrm{CH}_{3}\right), 2.12\left(\mathrm{~s}, 3 \mathrm{H}, \mathrm{CH}_{3}\right), 5.49\left(\mathrm{~s}, 2 \mathrm{H}, \mathrm{CH}_{2} \mathrm{O}\right), 6.13$ (s, 2H, $\left.\mathrm{NCH}_{2}\right), 6.80$ (s, 1H, Ar-H), 6.87 (d, $J=8.0 \mathrm{~Hz}, 1 \mathrm{H}$, Ar-H), 7.04 (d, J=8.0 Hz, 1H, Ar-H), $7.14 \sim 7.32$ (m, 6H, BZM- $\left.\mathrm{H}_{5}, \mathrm{H}_{6}, \mathrm{Ar}-\mathrm{H} \times 4\right), 7.34 \sim 7.48\left(\mathrm{~m}, 6 \mathrm{H}, \mathrm{Ar}^{\prime}-\mathrm{H}\right), 7.68$ (d, $\left.J=7.2 \mathrm{~Hz}, 1 \mathrm{H}, \mathrm{BZM}-\mathrm{H}_{7}\right), 7.71(\mathrm{~d}, J=7.6 \mathrm{~Hz}, 1 \mathrm{H}$, BZM-H $)$; IR (KBr) v: 3062, 3030, 2948, 2863, 1615 (W), 1602, 1507, 1464, 1219, $1023 \mathrm{~cm}^{-1}$. Anal. calcd for $\mathrm{C}_{32} \mathrm{H}_{27} \mathrm{~N}_{5} \mathrm{O}$ : C 77.24, H 5.47, N 14.07; found $\mathrm{C} 77.05, \mathrm{H}$ 5.60, N 13.92 .

3-[2-(2-甲氧基苯氧甲基)苯并咪唑-1-亚甲基]-5,6二苯基-1,2,4-三嗪(5f): 苂光黄色晶体, 收率 72\%. m.p. 90.7 91.5 ${ }^{\circ} \mathrm{C} ;{ }^{1} \mathrm{H}$ NMR (400 MHz, DMSO- $d_{6}$ ) $\delta: 3.57$ (s, $\left.3 \mathrm{H}, \mathrm{CH}_{3} \mathrm{O}\right), 5.50\left(\mathrm{~s}, 2 \mathrm{H}, \mathrm{CH}_{2} \mathrm{O}\right), 6.15\left(\mathrm{~s}, 2 \mathrm{H}, \mathrm{NCH}_{2}\right)$, $6.78 \sim 6.92(\mathrm{~m}, 3 \mathrm{H}, \mathrm{Ar}-\mathrm{H}), 7.18(\mathrm{~d}, J=7.6 \mathrm{~Hz}, 1 \mathrm{H}, \mathrm{Ar}-\mathrm{H})$, $7.21 \sim 7.33\left(\mathrm{~m}, 6 \mathrm{H}, \mathrm{BZM}-\mathrm{H}_{5}, \mathrm{H}_{6}, \mathrm{Ar}-\mathrm{H} \times 4\right), 7.36 \sim 7.48$ (m, 6H, Ar'-H), 7.68 (d, J=6.8 Hz, 1H, BZM-H $), 7.70$ (d, $J=7.6 \mathrm{~Hz}, 1 \mathrm{H}, \mathrm{BZM}-\mathrm{H}_{4}$ ); IR (KBr) v: 3062, 2951, 2834, 1618 (W), 1592, 1503, 1464, 1252, $1026 \mathrm{~cm}^{-1}$. Anal. calcd for $\mathrm{C}_{31} \mathrm{H}_{25} \mathrm{~N}_{5} \mathrm{O}_{2}$ : C 74.53, H 5.04, $\mathrm{N} \mathrm{14.02;} \mathrm{found} \mathrm{C} 74.28$, $\mathrm{H} 4.87, \mathrm{~N} 14.23$.

3-[2-(4-甲氧基苯氧甲基)苯并咪唑-1-亚甲基]-5,6二苯基-1,2,4-三嗪(5g): 荧光黄色晶体, 收率 79\%. m.p. $165.7 \sim 166.1{ }^{\circ} \mathrm{C} ;{ }^{1} \mathrm{H}$ NMR (500 MHz, DMSO- $\left.d_{6}\right) \delta: 3.61$ $\left(\mathrm{s}, 3 \mathrm{H}, \mathrm{OCH}_{3}\right), 5.46\left(\mathrm{~s}, 2 \mathrm{H}, \mathrm{CH}_{2} \mathrm{O}\right), 6.11\left(\mathrm{~s}, 2 \mathrm{H}, \mathrm{NCH}_{2}\right)$, $6.72(\mathrm{~d}, J=9.3 \mathrm{~Hz}, 2 \mathrm{H}, \operatorname{Ar}-\mathrm{H}), 6.76$ (d, $J=9.3 \mathrm{~Hz}, 2 \mathrm{H}$,
Ar-H), $7.21 \sim 7.32\left(\mathrm{~m}, 6 \mathrm{H}, \mathrm{BZM}-\mathrm{H}_{5}, \mathrm{H}_{6}, \mathrm{Ar}-\mathrm{H} \times 4\right), 7.38 \sim$ $7.48(\mathrm{~m}, 6 \mathrm{H}, \mathrm{Ar}-\mathrm{H}), 7.68 \sim 7.73\left(\mathrm{~m}, 2 \mathrm{H}, \mathrm{BZM}-\mathrm{H}_{4}, \mathrm{H}_{7}\right)$; IR (KBr) v: 3060, 2954, 2843, 1618 (W), 1506, 1455, 1236, $1036 \mathrm{~cm}^{-1}$. Anal. calcd for $\mathrm{C}_{31} \mathrm{H}_{25} \mathrm{~N}_{5} \mathrm{O}_{2}$ : C 74.53, H 5.04, N 14.02; found C 74.67, H 5.15, N 13.84 .

3-[2-(4-碘苯氧甲基)苯并咪唑-1-亚甲基]-5,6-二苯 基-1,2,4-三嗪(5h)：黄色晶体，收率 70\%. m.p. 181.0 $182.0{ }^{\circ} \mathrm{C} ;{ }^{1} \mathrm{H}$ NMR (400 MHz, DMSO- $\left.d_{6}\right) \delta: 5.64$ (s, 2H, $\left.\mathrm{CH}_{2} \mathrm{O}\right), 6.22\left(\mathrm{~s}, 2 \mathrm{H}, \mathrm{NCH}_{2}\right), 6.77(\mathrm{~d}, J=8.4 \mathrm{~Hz}, 2 \mathrm{H}$, Ar-H), $7.30 \sim 7.44\left(\mathrm{~m}, 6 \mathrm{H}, \mathrm{BZM}-\mathrm{H}_{5}, \mathrm{H}_{6}, \mathrm{Ar}^{\prime}-\mathrm{H} \times 4\right), 7.48 \sim$ 7.54 (m, 6H, Ar'-H), 7.57 (d, J=8.8 Hz, 2H, Ar-H), 7.82 (d, $J=7.2 \mathrm{~Hz}, 2 \mathrm{H}, \mathrm{BZM}-\mathrm{H}_{4}, \mathrm{H}_{7}$ ); IR (KBr) v: 3059, 2932, 2853, 1618 (W), 1582, 1507, 1484, 1461, 1236, 1023 $\mathrm{cm}^{-1}$. Anal. calcd for $\mathrm{C}_{30} \mathrm{H}_{22} \mathrm{IN}_{5} \mathrm{O}: \mathrm{C} 60.51, \mathrm{H} 3.72, \mathrm{~N}$ 11.76; found C 60.39, H 3.88, N 11.56.

3-[2-(2-溴苯氧甲基)苯并咪唑-1-亚甲基]-5,6-二苯 基-1,2,4-三嗪(5i)：黄色晶体，收率 73\%. m.p. 155.9 $157.2{ }^{\circ} \mathrm{C} ;{ }^{1} \mathrm{H}$ NMR (400 MHz, DMSO- $\left.d_{6}\right) \delta: 5.66$ (s, 2H, $\mathrm{CH}_{2} \mathrm{O}$ ), 6.19 (s, 2H, $\mathrm{NCH}_{2}$ ), 6.83 (t, $\left.J=7.6 \mathrm{~Hz}, 1 \mathrm{H}, \mathrm{Ar}-\mathrm{H}\right)$, $7.19(\mathrm{~d}, J=7.6 \mathrm{~Hz}, 2 \mathrm{H}, \mathrm{Ar}-\mathrm{H}), 7.22 \sim 7.35(\mathrm{~m}, 7 \mathrm{H}$, BZM- $\left.{ }_{5}, \mathrm{H}_{6}, \mathrm{Ar}-\mathrm{H}, \mathrm{Ar}-\mathrm{H} \times 4\right), 7.35 \sim 7.50$ (m, 6H, Ar'-H), 7.69 (d, $\left.J=7.6 \mathrm{~Hz}, 1 \mathrm{H}, \mathrm{BZM}-\mathrm{H}_{7}\right), 7.74$ (d, $J=7.6 \mathrm{~Hz}, 1 \mathrm{H}$, BZM-H 4 ); IR (KBr) v: 3059, 3030, 2954, 2866, 1615(W), $1585,1503,1480,1461,1242,1030 \mathrm{~cm}^{-1}$. Anal. calcd for $\mathrm{C}_{30} \mathrm{H}_{22} \mathrm{BrN}_{5} \mathrm{O}$ : C 65.70, $\mathrm{H}$ 4.04, N 12.77; found $\mathrm{C} 65.81, \mathrm{H}$ 3.90, N 12.94 .

3-[2-(2-氯苯氧甲基)苯并咪唑-1-亚甲基]-5,6-二苯 基-1,2,4-三嗪(5j)：黄色晶体，收率 77\%. m.p. 170.0 $171.0{ }^{\circ} \mathrm{C} ;{ }^{1} \mathrm{H}$ NMR (500 MHz, DMSO- $\left.d_{6}\right) \delta: 5.65$ (s, 2H, $\left.\mathrm{CH}_{2} \mathrm{O}\right), 6.18$ (s, 2H, $\left.\mathrm{NCH}_{2}\right), 6.89$ (t, $\left.J=7.5 \mathrm{~Hz}, 1 \mathrm{H}, \mathrm{Ar}-\mathrm{H}\right)$, $7.18(\mathrm{~d}, J=8.0 \mathrm{~Hz}, 2 \mathrm{H}, \operatorname{Ar}-\mathrm{H}), 7.24 \sim 7.32(\mathrm{~m}, 6 \mathrm{H}$, $\left.\mathrm{BZM}-\mathrm{H}_{5}, \mathrm{H}_{6}, \quad \mathrm{Ar}-\mathrm{H} \times 4\right), 7.37 \sim 7.48(\mathrm{~m}, 7 \mathrm{H}, \quad \mathrm{Ar}-\mathrm{H}$, Ar'-H×6), 7.70 (d, $\left.J=7.5 \mathrm{~Hz}, 1 \mathrm{H}, \mathrm{BZM}-\mathrm{H}_{7}\right), 7.73$ (d, $J=$ $\left.8.0 \mathrm{~Hz}, 1 \mathrm{H}, \mathrm{BZM}-\mathrm{H}_{4}\right)$; IR (KBr) v: 3059, 3030, 2958, 2870, 1618 (W), 1592, 1503, 1484, 1461, 1242, 1059 $\mathrm{cm}^{-1}$. Anal. calcd for $\mathrm{C}_{30} \mathrm{H}_{22} \mathrm{ClN}_{5} \mathrm{O}$ : C 71.49, $\mathrm{H} 4.40, \mathrm{~N}$ 13.90; found C 71.73, H 4.21, N 14.13.

3-[2-(3-氯苯氧甲基)苯并咪唑-1-亚甲基]-5,6-二苯 基-1,2,4-三嗪(5k)：黄色晶体，收率 76\%. m.p. 151.0 $151.5{ }^{\circ} \mathrm{C} ;{ }^{1} \mathrm{H}$ NMR (400 MHz, DMSO- $\left.d_{6}\right): \delta: 5.57$ (s, 2H, $\left.\mathrm{CH}_{2} \mathrm{O}\right), 6.13\left(\mathrm{~s}, 2 \mathrm{H}, \mathrm{NCH}_{2}\right), 6.81(\mathrm{~d}, J=8.0 \mathrm{~Hz}, 1 \mathrm{H}$, Ar-H), 6.90 (s, 1H, Ar-H), 6.94 (d, J=8.0 Hz, 1H, Ar-H), $7.20(\mathrm{t}, J=8.0 \mathrm{~Hz}, 1 \mathrm{H}, \mathrm{Ar}-\mathrm{H}), 7.24 \sim 7.34(\mathrm{~m}, 6 \mathrm{H}$, $\left.\mathrm{BZM}-\mathrm{H}_{5}, \mathrm{H}_{6}, \mathrm{Ar}^{\prime}-\mathrm{H} \times 4\right), 7.38 \sim 7.50\left(\mathrm{~m}, 6 \mathrm{H}, \mathrm{Ar}^{\prime}-\mathrm{H}\right), 7.73$ (d, $J=8.4 \mathrm{~Hz}, 2 \mathrm{H}, \mathrm{BZM}-\mathrm{H}_{4}, \mathrm{H}_{7}$ ); IR (KBr) v: 3062, 3030, 
2961, 2870, 1618 (W), 1595, 1582, 1507, 1478, 1461, 1222, $1020 \mathrm{~cm}^{-1}$. Anal. calcd for $\mathrm{C}_{30} \mathrm{H}_{22} \mathrm{ClN}_{5} \mathrm{O}$ : C 71.49, $\mathrm{H}$ 4.40, N 13.90; found C 71.64, H 4.52, N 13.77.

3-[2-(4-氯苯氧甲基)苯并咪唑-1-亚甲基]-5,6-二苯 基-1,2,4-三嗪(51): 荧光黄色晶体，收率 78\%. m.p. $175.1 \sim 175.9{ }^{\circ} \mathrm{C} ;{ }^{1} \mathrm{H}$ NMR (500 MHz, DMSO- $d_{6}$ ) $\delta: 5.54$ (s, 2H, $\left.\mathrm{CH}_{2} \mathrm{O}\right), 6.11\left(\mathrm{~s}, 2 \mathrm{H}, \mathrm{NCH}_{2}\right), 6.85(\mathrm{~d}, J=9.0 \mathrm{~Hz}$, 2H, Ar-H), 7.20 (d, $J=9.0 \mathrm{~Hz}, 2 \mathrm{H}, \mathrm{Ar}-\mathrm{H}), 7.22 \sim 7.33(\mathrm{~m}$, $\left.6 \mathrm{H}, \mathrm{BZM}-\mathrm{H}_{5}, \mathrm{H}_{6}, \mathrm{Ar}-\mathrm{H} \times 4\right), 7.38 \sim 7.48\left(\mathrm{~m}, 6 \mathrm{H}, \mathrm{Ar}^{\prime}-\mathrm{H}\right)$, $7.71\left(\mathrm{~d}, J=8.4 \mathrm{~Hz}, 2 \mathrm{H}, \mathrm{BZM}-\mathrm{H}_{4}, \mathrm{H}_{7}\right)$; IR $(\mathrm{KBr}) v: 3059$, 2925, 2853, 1618 (W), 1595, 1491, 1461, 1239, 1010 $\mathrm{cm}^{-1}$. Anal. calcd for $\mathrm{C}_{30} \mathrm{H}_{22} \mathrm{ClN}_{5} \mathrm{O}: \mathrm{C} 71.49, \mathrm{H} 4.40, \mathrm{~N}$ 13.90; found C 71.32, H 4.25, N 14.11 .

3-[2-(4-硝基苯氧甲基)苯并咪唑-1-亚甲基]-5,6-二 苯基-1,2,4-三嗪 (5m): 黄色晶体, 收率 70\%. m.p. $192.0 \sim 192.9{ }^{\circ} \mathrm{C} ;{ }^{1} \mathrm{H}$ NMR (400 MHz, DMSO- $d_{6}$ ) $\delta: 5.74$ (s, 2H, $\left.\mathrm{CH}_{2} \mathrm{O}\right), 6.15\left(\mathrm{~s}, 2 \mathrm{H}, \mathrm{NCH}_{2}\right), 7.03(\mathrm{~d}, J=8.8 \mathrm{~Hz}$, $2 \mathrm{H}, \mathrm{Ar}-\mathrm{H}), 7.20 \sim 7.36\left(\mathrm{~m}, 6 \mathrm{H}, \mathrm{BZM}-\mathrm{H}_{5}, \mathrm{H}_{6}, \mathrm{Ar}-\mathrm{H} \times 4\right)$, $7.36 \sim 7.50\left(\mathrm{~m}, 6 \mathrm{H}, \mathrm{Ar}^{\prime}-\mathrm{H}\right), 7.73\left(\mathrm{~m}, 2 \mathrm{H}, \mathrm{BZM}-\mathrm{H}_{4}, \mathrm{H}_{7}\right)$, 8.05 (d, $J=9.6 \mathrm{~Hz}, 2 \mathrm{H}, \mathrm{Ar}-\mathrm{H})$; IR (KBr) v: 3059, 2932, 2850, $1611(\mathrm{~W}), 1592,1510,1494,1464,1340,1252$, $1108 \mathrm{~cm}^{-1}$. Anal. calcd for $\mathrm{C}_{30} \mathrm{H}_{22} \mathrm{~N}_{6} \mathrm{O}_{3}: \mathrm{C} 70.03, \mathrm{H} 4.31$, N 16.33; found C 69.76, H 4.08, N 16.17.

3-[2-(1-萗氧甲基)苯并咪唑-1-亚甲基]-5,6-二苯基1,2,4-三嗪(5n): 黄色晶体, 收率 74\%. m.p. 171.5 $172.5{ }^{\circ} \mathrm{C} ;{ }^{1} \mathrm{H}$ NMR (400 MHz, DMSO- $\left.d_{6}\right) \delta: 5.76(\mathrm{~s}, 2 \mathrm{H}$, $\left.\mathrm{CH}_{2} \mathrm{O}\right), 6.21\left(\mathrm{~s}, 2 \mathrm{H}, \mathrm{NCH}_{2}\right), 7.01(\mathrm{~d}, J=7.6 \mathrm{~Hz}, 2 \mathrm{H}$, Ar-H), $7.11 \sim 7.45\left(\mathrm{~m}, \quad 15 \mathrm{H}, \quad\right.$ BZM- $\mathrm{H}_{5}, \mathrm{H}_{6}, \quad \mathrm{Ar}-\mathrm{H} \times 3$, Ar'-H×10), 7.48 (d, $J=8.4 \mathrm{~Hz}, 1 \mathrm{H}, \mathrm{Ar}-\mathrm{H}), 7.69$ (d, $J=8.8$ $\mathrm{Hz}, 1 \mathrm{H}, \mathrm{Ar}-\mathrm{H}$ ), 7.77 (d, $J=7.6 \mathrm{~Hz}, 2 \mathrm{H}, \mathrm{BZM}-\mathrm{H}_{4}, \mathrm{H}_{7}$ ); IR (KBr) v: 3056, 2944, 2857, $1618(\mathrm{~W}), 1598,1579,1507$, 1461, 1268, $1098 \mathrm{~cm}^{-1}$. Anal. calcd for $\mathrm{C}_{34} \mathrm{H}_{25} \mathrm{~N}_{5} \mathrm{O}: \mathrm{C}$ 78.59, H 4.85, N 13.48; found C 78.75, H 4.72, N 13.67.

3-[2-(2-菜氧甲基)苯并咪唑-1-亚甲基]-5,6-二苯基1,2,4-三嗪(5o): 浅黄色晶体, 收率 75\%. m.p. 147.4 $148.4{ }^{\circ} \mathrm{C} ;{ }^{1} \mathrm{H}$ NMR (400 MHz, DMSO- $\left.d_{6}\right) \delta: 5.66$ (s, $2 \mathrm{H}$, $\left.\mathrm{CH}_{2} \mathrm{O}\right), 6.16\left(\mathrm{~s}, 2 \mathrm{H}, \mathrm{NCH}_{2}\right), 6.83(\mathrm{~d}, J=8.0 \mathrm{~Hz}, 1 \mathrm{H}$, Ar-H), $7.10 \sim 7.24(\mathrm{~m}, 3 \mathrm{H}$, Ar-H), $7.24 \sim 7.48(\mathrm{~m}, 12 \mathrm{H}$, $\left.\mathrm{BZM}-\mathrm{H}_{5}, \mathrm{H}_{6}, \mathrm{Ar}-\mathrm{H} \times 10\right), 7.60 \sim 7.80\left(\mathrm{~m}, 5 \mathrm{H}, \mathrm{BZM}-\mathrm{H}_{4}, \mathrm{H}_{7}\right.$, $\mathrm{Ar}-\mathrm{H} \times 3$ ); IR (KBr) v: 3056, 2942, 2857, 1631 (W), 1602, $1510,1464,1255,1010 \mathrm{~cm}^{-1}$. Anal. calcd for $\mathrm{C}_{34} \mathrm{H}_{25} \mathrm{~N}_{5} \mathrm{O}$ : C 78.59, H 4.85, N 13.48; found C 78.33, H 4.96, N 13.24.

3-[2-(苯氧甲基)苯并咪唑-1-亚甲基]-5,6-二(4-甲氧 基苯基)-1,2,4-三嗪(6a): 黄色晶体, 收率 72\%. m.p. $143.8 \sim 145.3{ }^{\circ} \mathrm{C} ;{ }^{1} \mathrm{H}$ NMR (400 MHz, DMSO- $d_{6}$ ) $\delta: 3.75$ (s, $\left.3 \mathrm{H}, \mathrm{CH}_{3} \mathrm{O}\right), 3.79$ (s, $\left.3 \mathrm{H}, \mathrm{CH}_{3} \mathrm{O}\right), 5.52\left(\mathrm{~s}, 2 \mathrm{H}, \mathrm{CH}_{2} \mathrm{O}\right)$, $6.06\left(\mathrm{~s}, 2 \mathrm{H}, \mathrm{NCH}_{2}\right), 6.80 \sim 6.90(\mathrm{~m}, 5 \mathrm{H}, \mathrm{Ar}-\mathrm{H} \times 3$, Ar'-H×2), 6.99 (d, $\left.J=8.8 \mathrm{~Hz}, 2 \mathrm{H}, \mathrm{Ar}^{\prime}-\mathrm{H}\right), 7.19$ (t, $J=7.8$ $\mathrm{Hz}, \quad 2 \mathrm{H}, \quad$ Ar-H), $7.22 \sim 7.32\left(\mathrm{~m}, \quad 4 \mathrm{H}, \quad\right.$ BZM- $\mathrm{H}_{5}, \mathrm{H}_{6}$, Ar'-H×2), 7.38 (d, $J=8.8$ Hz, 2H, Ar'-H), 7.71 (d, $J=7.6$ $\mathrm{Hz}, 2 \mathrm{H}, \mathrm{BZM}-\mathrm{H}_{4}, \mathrm{H}_{7}$ ); IR (KBr) v: 3059, 2935, 2843, 1605 (S), 1497, 1461, 1252, $1026 \mathrm{~cm}^{-1}$. Anal. calcd for $\mathrm{C}_{32} \mathrm{H}_{27}$ $\mathrm{N}_{5} \mathrm{O}_{3}$ : C 72.57, H 5.14, N 13.22; found C 72.43, H 5.32, N 13.42.

3-[2-(2-甲基苯氧甲基)苯并咪唑-1-亚甲基]-5,6-二 (4-甲氧基苯基)-1,2,4-三嗪(6b): 黄色晶体，收率 73\%. m.p. $155.2 \sim 156.9{ }^{\circ} \mathrm{C} ;{ }^{1} \mathrm{H}$ NMR (500 MHz, DMSO- $d_{6}$ ) $\delta$ : 1.77 (s, $\left.3 \mathrm{H}, \mathrm{CH}_{3}\right), 3.74$ (s, $\left.3 \mathrm{H}, \mathrm{CH}_{3} \mathrm{O}\right), 3.79$ (s, $3 \mathrm{H}, \mathrm{CH}_{3} \mathrm{O}$ ), $5.53\left(\mathrm{~s}, 2 \mathrm{H}, \mathrm{CH}_{2} \mathrm{O}\right), 6.08\left(\mathrm{~s}, 2 \mathrm{H}, \mathrm{NCH}_{2}\right), 6.80(\mathrm{t}, J=7.3$ $\mathrm{Hz}, 1 \mathrm{H}, \mathrm{Ar}-\mathrm{H}), 6.83$ (d, $\left.J=8.5 \mathrm{~Hz}, 2 \mathrm{H}, \mathrm{Ar}^{\prime}-\mathrm{H}\right), 6.98$ (d, $\left.J=9.0 \mathrm{~Hz}, 2 \mathrm{H}, \mathrm{Ar}^{\prime}-\mathrm{H}\right), 7.01(\mathrm{~d}, J=8.0 \mathrm{~Hz}, 1 \mathrm{H}, \operatorname{Ar}-\mathrm{H})$, $7.11(\mathrm{t}, J=7.5 \mathrm{~Hz}, 1 \mathrm{H}, \operatorname{Ar}-\mathrm{H}), 7.20(\mathrm{~d}, J=9.0 \mathrm{~Hz}, 3 \mathrm{H}$, Ar-H, Ar'-H×2), 7.23 7.30 (m, 2H, BZM- $\left.\mathrm{H}_{5}, \mathrm{H}_{6}\right), 7.35$ (d, $\left.J=9.0 \mathrm{~Hz}, 2 \mathrm{H}, \mathrm{Ar}^{\prime}-\mathrm{H}\right), 7.67(\mathrm{~d}, J=7.0 \mathrm{~Hz}, 1 \mathrm{H}$, BZM- $\left.H_{7}\right), 7.72\left(\mathrm{~d}, J=7.0 \mathrm{~Hz}, 1 \mathrm{H}, \mathrm{BZM}-\mathrm{H}_{4}\right)$; IR $(\mathrm{KBr}) v$ : 3056, 3010, 2938, 2840, 1607 (S), 1578, 1497, 1464, 1255, $1030 \mathrm{~cm}^{-1}$. Anal. calcd for $\mathrm{C}_{33} \mathrm{H}_{29} \mathrm{~N}_{5} \mathrm{O}_{3}: \mathrm{C} 72.91$, H 5.38, N 12.88; found C 73.10, H 5.22, N 12.76.

3-[2-(3-甲基苯氧甲基)苯并咪唑-1-亚甲基]-5,6-二 (4-甲氧基苯基)-1,2,4-三嗪(6c): 黄色晶体, 收率 70\%. m.p. $165.3 \sim 166.2{ }^{\circ} \mathrm{C} ;{ }^{1} \mathrm{H}$ NMR $\left(400 \mathrm{MHz}\right.$, DMSO- $\left.d_{6}\right) \delta$ : $2.15\left(\mathrm{~s}, 3 \mathrm{H}, \mathrm{CH}_{3}\right), 3.75\left(\mathrm{~s}, 3 \mathrm{H}, \mathrm{CH}_{3} \mathrm{O}\right), 3.78\left(\mathrm{~s}, 3 \mathrm{H}, \mathrm{CH}_{3} \mathrm{O}\right)$, $5.48\left(\mathrm{~s}, 2 \mathrm{H}, \mathrm{CH}_{2} \mathrm{O}\right), 6.05\left(\mathrm{~s}, 2 \mathrm{H}, \mathrm{NCH}_{2}\right), 6.60(\mathrm{~s}, 1 \mathrm{H}$, Ar-H), 6.66 (d, $J=7.2 \mathrm{~Hz}, 1 \mathrm{H}, \mathrm{Ar}-\mathrm{H}), 6.70$ (d, $J=7.6 \mathrm{~Hz}$, 1H, Ar-H), 6.87 (d, $\left.J=8.8 \mathrm{~Hz}, 2 \mathrm{H}, \mathrm{Ar}^{\prime}-\mathrm{H}\right), 6.99$ (d, $J=9.2$ $\mathrm{Hz}, 2 \mathrm{H}, \mathrm{Ar}-\mathrm{H}), 7.06$ (t, $J=7.8 \mathrm{~Hz}, 1 \mathrm{H}, \mathrm{Ar}-\mathrm{H}), 7.27$ (d, $J=$ $8.8 \mathrm{~Hz}, 2 \mathrm{H}, \mathrm{Ar}-\mathrm{H}), 7.22 \sim 7.32$ (m, 2H, BZM- $\left.\mathrm{H}_{5}, \mathrm{H}_{6}\right), 7.38$ (d, $\left.J=8.8 \mathrm{~Hz}, 2 \mathrm{H}, \operatorname{Ar}^{\prime}-\mathrm{H}\right), 7.71$ (d, $J=8.4 \mathrm{~Hz}, 2 \mathrm{H}$, BZM- $\mathrm{H}_{4}, \mathrm{H}_{7}$ ); IR ( $\left.\mathrm{KBr}\right)$ v: 3065, 2940, 2842, 1609 (S), $1495,1459,1257,1029 \mathrm{~cm}^{-1}$. Anal. calcd for $\mathrm{C}_{33} \mathrm{H}_{29} \mathrm{~N}_{5} \mathrm{O}_{3}$ : C 72.91, H 5.38, N 12.88; found C 72.68, H 5.59, N 12.71.

3-[2-(4-甲基苯氧甲基)苯并咪唑-1-亚甲基]-5,6-二 (4-甲氧基苯基)-1,2,4-三嗪(6d): 浅黄色晶体，收率 81\%. m.p. $128.6 \sim 129.3{ }^{\circ} \mathrm{C} ;{ }^{1} \mathrm{H}$ NMR (500 MHz, DMSO- $\left.d_{6}\right) \delta$ : 2.15 (s, $\left.3 \mathrm{H}, \mathrm{CH}_{3}\right), 3.75$ (s, $\left.3 \mathrm{H}, \mathrm{CH}_{3} \mathrm{O}\right), 3.79$ (s, $3 \mathrm{H}, \mathrm{CH}_{3} \mathrm{O}$ ), $5.47\left(\mathrm{~s}, 2 \mathrm{H}, \mathrm{CH}_{2} \mathrm{O}\right), 6.04$ (s, $\left.2 \mathrm{H}, \mathrm{NCH}_{2}\right), 6.72(\mathrm{~d}, J=8.5$ $\mathrm{Hz}, 2 \mathrm{H}, \mathrm{Ar}-\mathrm{H}), 6.86$ (d, J=9.0 Hz, 2H, Ar'-H), 6.96 (d, $J=8.5 \mathrm{~Hz}, 2 \mathrm{H}, \mathrm{Ar}-\mathrm{H}), 6.99$ (d, $\left.J=8.5 \mathrm{~Hz}, 2 \mathrm{H}, \mathrm{Ar}^{\prime}-\mathrm{H}\right)$, $7.24\left(\mathrm{~d}, J=8.5 \mathrm{~Hz}, 2 \mathrm{H}, \mathrm{Ar}^{\prime}-\mathrm{H}\right), 7.21 \sim 7.31(\mathrm{~m}, 2 \mathrm{H}$, BZM- $\left.\mathrm{H}_{5}, \mathrm{H}_{6}\right), 7.38$ (d, $\left.J=8.5 \mathrm{~Hz}, 2 \mathrm{H}, \mathrm{Ar}-\mathrm{H}\right), 7.69$ (d, $J=$ 
$\left.8.0 \mathrm{~Hz}, 1 \mathrm{H}, \mathrm{BZM}-\mathrm{H}_{7}\right), 7.70$ (d, $\left.J=8.0 \mathrm{~Hz}, 1 \mathrm{H}, \mathrm{BZM}-\mathrm{H}_{4}\right)$; IR (KBr) v: 3062, 2938, 2840, 1608 (S), 1579, 1497, 1461, $1259,1033 \mathrm{~cm}^{-1}$. Anal. calcd for $\mathrm{C}_{33} \mathrm{H}_{29} \mathrm{~N}_{5} \mathrm{O}_{3}: \mathrm{C} 72.91, \mathrm{H}$ 5.38, N 12.88; found C 73.19, H 5.48, N 13.10.

3-[2-(2,4-二甲基苯氧甲基)苯并咪唑-1-亚甲基]-5,6二(4-甲氧基苯基)-1,2,4-三嗪(6e): 黄色晶体, 收率 71\%. m.p. $62.7 \sim 63.4{ }^{\circ} \mathrm{C} ;{ }^{1} \mathrm{H}$ NMR (400 MHz, DMSO-d $\left.d_{6}\right) \delta$ : $1.73\left(\mathrm{~s}, 3 \mathrm{H}, \mathrm{CH}_{3}\right), 2.12\left(\mathrm{~s}, 3 \mathrm{H}, \mathrm{CH}_{3}\right), 3.74\left(\mathrm{~s}, 3 \mathrm{H}, \mathrm{CH}_{3} \mathrm{O}\right)$, $3.79\left(\mathrm{~s}, 3 \mathrm{H}, \mathrm{CH}_{3} \mathrm{O}\right), 5.48\left(\mathrm{~s}, 2 \mathrm{H}, \mathrm{CH}_{2} \mathrm{O}\right), 6.07(\mathrm{~s}, 2 \mathrm{H}$, $\left.\mathrm{NCH}_{2}\right), 6.79$ (s, 1H, Ar-H), 6.83 (d, J=8.4 Hz, 2H, Ar'-H), $6.88(\mathrm{~d}, J=8.4 \mathrm{~Hz}, 1 \mathrm{H}, \mathrm{Ar}-\mathrm{H}), 6.99$ (d, $J=8.8 \mathrm{~Hz}, 2 \mathrm{H}$, Ar'-H), 7.05 (d, $J=8.4 \mathrm{~Hz}, 1 \mathrm{H}, \mathrm{Ar}-\mathrm{H}), 7.18$ (d, $J=9.0 \mathrm{~Hz}$, $\left.2 \mathrm{H}, \mathrm{Ar}^{\prime}-\mathrm{H}\right), 7.22 \sim 7.32\left(\mathrm{~m}, 2 \mathrm{H}, \mathrm{BZM}-\mathrm{H}_{5}, \mathrm{H}_{6}\right), 7.35$ (d, $J=$ $8.8 \mathrm{~Hz}, 2 \mathrm{H}, \mathrm{Ar}^{\prime}-\mathrm{H}$ ), 7.67 (d, $J=8.0 \mathrm{~Hz}, 1 \mathrm{H}, \mathrm{BZM}-\mathrm{H}_{7}$ ), 7.72 (d, $\left.J=7.2 \mathrm{~Hz}, 1 \mathrm{H}, \mathrm{BZM}-\mathrm{H}_{4}\right)$; IR (KBr) $v$ : 3062, 2935, 2840, 1608 (S), 1579, 1500, 1461, 1255, $1030 \mathrm{~cm}^{-1}$. Anal. calcd for $\mathrm{C}_{34} \mathrm{H}_{31} \mathrm{~N}_{5} \mathrm{O}_{3}$ : C 73.23, H 5.60, N 12.56; found $\mathrm{C}$ 73.12, H 5.41, N 12.71 .

3-[2-(2-甲氧基苯氧甲基)苯并咪唑-1-亚甲基]-5,6二(4-甲氧基苯基)-1,2,4-三嗪(6f): 黄色晶体，收率 71\%. m.p. $162.6 \sim 164.0{ }^{\circ} \mathrm{C} ;{ }^{1} \mathrm{H}$ NMR (400 MHz, DMSO- $\left.d_{6}\right) \delta$ : $3.56\left(\mathrm{~s}, 3 \mathrm{H}, \mathrm{OCH}_{3}\right), 3.75\left(\mathrm{~s}, 3 \mathrm{H}, \mathrm{CH}_{3} \mathrm{O}\right), 3.78(\mathrm{~s}, 3 \mathrm{H}$, $\left.\mathrm{CH}_{3} \mathrm{O}\right), 5.49\left(\mathrm{~s}, 2 \mathrm{H}, \mathrm{CH}_{2} \mathrm{O}\right), 6.09\left(\mathrm{~s}, 2 \mathrm{H}, \mathrm{NCH}_{2}\right), 6.80 \sim$ $6.90\left(\mathrm{~m}, 5 \mathrm{H}, \mathrm{Ar}-\mathrm{H} \times 3, \mathrm{Ar}^{\prime}-\mathrm{H} \times 2\right), 6.98(\mathrm{~d}, J=8.8 \mathrm{~Hz}, 2 \mathrm{H}$, Ar'-H), 7.18 (d, $J=8.0 \mathrm{~Hz}, 1 \mathrm{H}, \mathrm{Ar}-\mathrm{H}), 7.72$ (d, $J=9.2 \mathrm{~Hz}$, $2 \mathrm{H}, \mathrm{Ar}-\mathrm{H}), 7.22 \sim 7.30$ (m, 2H, BZM-H $\left.{ }_{5} \mathrm{H}_{6}\right), 7.39$ (d, $J=$ $\left.8.8 \mathrm{~Hz}, 2 \mathrm{H}, \mathrm{Ar}^{\prime}-\mathrm{H}\right), 7.67$ (d, $\left.J=8.4 \mathrm{~Hz}, 1 \mathrm{H}, \mathrm{BZM}-\mathrm{H}_{7}\right), 7.70$ (d, $J=7.2 \mathrm{~Hz}, 1 \mathrm{H}, \mathrm{BZM}-\mathrm{H}_{4}$ ); IR (KBr) $v$ : 3066, 2938, 2837, 1608 (S), 1579, 1503, 1461, 1255, $1026 \mathrm{~cm}^{-1}$. Anal. calcd for $\mathrm{C}_{33} \mathrm{H}_{29} \mathrm{~N}_{5} \mathrm{O}_{4}$ : C 70.83, H 5.22, N 12.51; found $\mathrm{C}$ 70.67, H 5.47, N 12.69.

3-[2-(4-甲氧基苯氧甲基)苯并咪唑-1-亚甲基]-5,6二(4-甲氧基苯基)-1,2,4-三嗪(6g): 黄色晶体, 收率 76\%. m.p. $128.7 \sim 130.0{ }^{\circ} \mathrm{C} ;{ }^{1} \mathrm{H}$ NMR (400 MHz, DMSO- $\left.d_{6}\right) \delta$ : $3.62\left(\mathrm{~s}, 3 \mathrm{H}, \mathrm{CH}_{3} \mathrm{O}\right), 3.75\left(\mathrm{~s}, 3 \mathrm{H}, \mathrm{CH}_{3} \mathrm{O}\right), 3.79(\mathrm{~s}, 3 \mathrm{H}$, $\left.\mathrm{CH}_{3} \mathrm{O}\right), 5.45\left(\mathrm{~s}, 2 \mathrm{H}, \mathrm{CH}_{2} \mathrm{O}\right), 6.05\left(\mathrm{~s}, 2 \mathrm{H}, \mathrm{NCH}_{2}\right), 6.73$ (d, $J=8.8 \mathrm{~Hz}, 2 \mathrm{H}, \mathrm{Ar}-\mathrm{H}), 6.78$ (d, $J=8.8 \mathrm{~Hz}, 2 \mathrm{H}, \mathrm{Ar}-\mathrm{H}), 6.86$ (d, $\left.J=8.8 \mathrm{~Hz}, 2 \mathrm{H}, \mathrm{Ar}^{\prime}-\mathrm{H}\right), 6.99$ (d, $\left.J=8.8 \mathrm{~Hz}, 2 \mathrm{H}, \mathrm{Ar}^{\prime}-\mathrm{H}\right)$, $7.25\left(\mathrm{~d}, J=8.8 \mathrm{~Hz}, 2 \mathrm{H}, \mathrm{Ar}^{\prime}-\mathrm{H}\right), 7.20 \sim 7.32(\mathrm{~m}, 2 \mathrm{H}$, BZM-H $\left.{ }_{5}, \mathrm{H}_{6}\right), 7.39$ (d, $\left.J=8.8 \mathrm{~Hz}, 2 \mathrm{H}, \mathrm{Ar}^{\prime}-\mathrm{H}\right), 7.70$ (d, $J=$ $\left.7.2 \mathrm{~Hz}, 2 \mathrm{H}, \mathrm{BZM}-\mathrm{H}_{4}, \mathrm{H}_{7}\right)$; IR (KBr) v: 3062, 2935, 2840, 1608 (S), 1507, 1458, 1252, $1030 \mathrm{~cm}^{-1}$. Anal. calcd for $\mathrm{C}_{33} \mathrm{H}_{29} \mathrm{~N}_{5} \mathrm{O}_{4}$ : C 70.83, H 5.22, N 12.51; found C 70.96, H 5.13, N 12.40 .

3-[2-(4-碘苯氧甲基)苯并咪唑-1-亚甲基]-5,6-二(4- 甲氧基苯基)-1,2,4-三嗪(6h): 苂光黄色晶体，收率 70\%. m.p. 178.0 179.2 ${ }^{\circ} \mathrm{C}$; ${ }^{1} \mathrm{H}$ NMR (400 MHz, DMSO- $\left.d_{6}\right) \delta$ : $3.76\left(\mathrm{~s}, 3 \mathrm{H}, \mathrm{CH}_{3} \mathrm{O}\right), 3.79\left(\mathrm{~s}, 3 \mathrm{H}, \mathrm{CH}_{3} \mathrm{O}\right), 5.52(\mathrm{~s}, 2 \mathrm{H}$, $\left.\mathrm{CH}_{2} \mathrm{O}\right), 6.05\left(\mathrm{~s}, 2 \mathrm{H}, \mathrm{NCH}_{2}\right), 6.67(\mathrm{~d}, J=9.2 \mathrm{~Hz}, 2 \mathrm{H}$, Ar-H), 6.86 (d, $\left.J=8.8 \mathrm{~Hz}, 2 \mathrm{H}, \mathrm{Ar}^{\prime}-\mathrm{H}\right), 6.99$ (d, $J=8.8 \mathrm{~Hz}$, 2H, Ar'-H), 7.22 (d, J=9.2 Hz, 2H, Ar'-H), 7.22 $7.33(\mathrm{~m}$, 2H, BZM-H $\left.{ }_{5}, \mathrm{H}_{6}\right), 7.38$ (d, $\left.J=8.8 \mathrm{~Hz}, 2 \mathrm{H}, \mathrm{Ar}-\mathrm{H}\right), 7.46$ (d, $J=8.8 \mathrm{~Hz}, 2 \mathrm{H}, \mathrm{Ar}-\mathrm{H}), 7.66 \sim 7.74\left(\mathrm{~m}, 2 \mathrm{H}, \mathrm{BZM}-\mathrm{H}_{4}, \mathrm{H}_{7}\right)$; IR (KBr) v: 3052, 2932, 2840, 1608 (S), 1575, 1493, 1487, 1461, 1259, $1030 \mathrm{~cm}^{-1}$. Anal. calcd for $\mathrm{C}_{32} \mathrm{H}_{26} \mathrm{IN}_{5} \mathrm{O}_{3}: \mathrm{C}$ 58.63, H 4.00, N 10.68; found C 58.87, H 3.85, N 10.48.

3-[2-(2-溴苯氧甲基)苯并咪唑-1-亚甲基]-5,6-二(4甲氧基苯基)-1,2,4-三嗪(6i): 黄色晶体, 收率 69\%. m.p. $178.8 \sim 179.9{ }^{\circ} \mathrm{C} ;{ }^{1} \mathrm{H}$ NMR (400 MHz, DMSO- $\left.d_{6}\right) \delta: 3.74$ $\left(\mathrm{s}, 3 \mathrm{H}, \mathrm{CH}_{3} \mathrm{O}\right), 3.79\left(\mathrm{~s}, 3 \mathrm{H}, \mathrm{CH}_{3} \mathrm{O}\right), 5.65\left(\mathrm{~s}, 2 \mathrm{H}, \mathrm{CH}_{2} \mathrm{O}\right)$, $6.12\left(\mathrm{~s}, 2 \mathrm{H}, \mathrm{NCH}_{2}\right), 6.78 \sim 6.88(\mathrm{~m}, 3 \mathrm{H}, \mathrm{Ar}-\mathrm{H}), 6.99(\mathrm{~d}$, $\left.J=8.8 \mathrm{~Hz}, 2 \mathrm{H}, \mathrm{Ar}^{\prime}-\mathrm{H}\right), 7.17$ (d, $\left.J=8.8 \mathrm{~Hz}, 2 \mathrm{H}, \mathrm{Ar}^{\prime}-\mathrm{H}\right)$, $7.22 \sim 7.34\left(\mathrm{~m}, 3 \mathrm{H}, \mathrm{BZM}-\mathrm{H}_{5}, \mathrm{H}_{6}, \mathrm{Ar}-\mathrm{H}\right), 7.36(\mathrm{~d}, J=8.8$ $\left.\mathrm{Hz}, 2 \mathrm{H}, \mathrm{Ar}^{\prime}-\mathrm{H}\right), 7.42$ (d, J=7.6 Hz, 2H, Ar'-H), 7.67 (d, $\left.J=6.8 \mathrm{~Hz}, 1 \mathrm{H}, \mathrm{BZM}-\mathrm{H}_{7}\right), 7.74(\mathrm{~d}, J=6.8 \mathrm{~Hz}, 1 \mathrm{H}$, BZM-H $)_{4}$; IR (KBr) v: 3066, 2935, 2840, 1608 (S), 1579, 1497, 1480, 1461, 1255, $1030 \mathrm{~cm}^{-1}$. Anal. calcd for $\mathrm{C}_{32} \mathrm{H}_{26} \mathrm{BrN}_{5} \mathrm{O}_{3}$ : C 63.16, H 4.31, N 11.51; found C 62.97, $\mathrm{H} 4.08, \mathrm{~N} 11.78$.

3-[2-(2-氯苯氧甲基)苯并咪唑-1-亚甲基]-5,6-二(4甲氧基苯基)-1,2,4-三嗪(6j)：黄色晶体，收率 71\%. m.p. $169.9 \sim 170.7{ }^{\circ} \mathrm{C} ;{ }^{1} \mathrm{H}$ NMR (400 MHz, DMSO- $\left.d_{6}\right) \delta: 3.74$ (s, 3H, $\left.\mathrm{CH}_{3} \mathrm{O}\right), 3.79$ (s, 3H, $\left.\mathrm{CH}_{3} \mathrm{O}\right), 5.65$ (s, 2H, $\left.\mathrm{CH}_{2} \mathrm{O}\right)$, $6.12\left(\mathrm{~s}, 2 \mathrm{H}, \mathrm{NCH}_{2}\right), 6.83$ (d, J=8.8 Hz, 2H, Ar'-H), 6.89 (t, $J=7.8 \mathrm{~Hz}, 1 \mathrm{H}, \mathrm{Ar}-\mathrm{H}), 6.99$ (d, $\left.J=8.8 \mathrm{~Hz}, 2 \mathrm{H}, \mathrm{Ar}^{\prime}-\mathrm{H}\right)$, $7.17\left(\mathrm{~d}, J=8.8 \mathrm{~Hz}, 2 \mathrm{H}, \mathrm{Ar}^{\prime}-\mathrm{H}\right), 7.22 \sim 7.32(\mathrm{~m}, 4 \mathrm{H}$, BZM- $\left.H_{5}, \mathrm{H}_{6}, \mathrm{Ar}-\mathrm{H} \times 2\right), 7.36(\mathrm{~d}, J=8.8 \mathrm{~Hz}, 2 \mathrm{H}, \mathrm{Ar}-\mathrm{H})$, $7.43(\mathrm{~d}, J=8.4 \mathrm{~Hz}, 1 \mathrm{H}, \operatorname{Ar}-\mathrm{H}), 7.69$ (d, $J=7.8 \mathrm{~Hz}, 1 \mathrm{H}$, $\left.\mathrm{BZM}-\mathrm{H}_{7}\right), 7.74$ (d, $\left.J=7.2 \mathrm{~Hz}, 1 \mathrm{H}, \mathrm{BZM}-\mathrm{H}_{4}\right)$; IR (KBr) v: 3062, 2935, 2837, 1608(S), 1579, 1497, 1484, 1461, 1255, $1026 \mathrm{~cm}^{-1}$. Anal. calcd for $\mathrm{C}_{32} \mathrm{H}_{26} \mathrm{ClN}_{5} \mathrm{O}_{3}: \mathrm{C} 68.14, \mathrm{H}$ 4.65, N 12.42; found C 68.04, H 4.76, N 12.25.

3-[2-(3-氯苯氧甲基)苯并咪唑-1-亚甲基]-5,6-二(4甲氧基苯基)-1,2,4-三嗪(6k): 黄色晶体，收率 70\%. m.p. $182.9 \sim 184.3{ }^{\circ} \mathrm{C} ;{ }^{1} \mathrm{H}$ NMR (400 MHz, DMSO- $d_{6}$ ) $\delta: 3.75$ $\left(\mathrm{s}, 3 \mathrm{H}, \mathrm{CH}_{3} \mathrm{O}\right), 3.78\left(\mathrm{~s}, 3 \mathrm{H}, \mathrm{CH}_{3} \mathrm{O}\right), 5.56\left(\mathrm{~s}, 2 \mathrm{H}, \mathrm{CH}_{2} \mathrm{O}\right)$, 6.06 (s, 2H, $\left.\mathrm{NCH}_{2}\right), 6.81$ (d, $\left.J=8.8 \mathrm{~Hz}, 1 \mathrm{H}, \mathrm{Ar}-\mathrm{H}\right), 6.86$ (d, $\left.J=8.8 \mathrm{~Hz}, 2 \mathrm{H}, \mathrm{Ar}^{\prime}-\mathrm{H}\right), 6.89$ (s, 1H, Ar-H), 6.94 (d, $J=$ $8.0 \mathrm{~Hz}, 1 \mathrm{H}, \mathrm{Ar}-\mathrm{H}), 6.99$ (d, $\left.J=8.8 \mathrm{~Hz}, 2 \mathrm{H}, \mathrm{Ar}^{\prime}-\mathrm{H}\right), 7.20$ (t, $J=8.0 \mathrm{~Hz}, 1 \mathrm{H}, \mathrm{Ar}-\mathrm{H}), 7.27$ (d, $\left.J=8.8 \mathrm{~Hz}, 2 \mathrm{H}, \mathrm{Ar}^{\prime}-\mathrm{H}\right)$, 
$7.23 \sim 7.33\left(\mathrm{~m}, 2 \mathrm{H}, \mathrm{BZM}-\mathrm{H}_{5}, \mathrm{H}_{6}\right), 7.39(\mathrm{~d}, J=8.8 \mathrm{~Hz}, 2 \mathrm{H}$, Ar'-H), 7.71 (d, $\left.J=7.2 \mathrm{~Hz}, 1 \mathrm{H}, \mathrm{BZM}-\mathrm{H}_{7}\right), 7.72$ (d, $J=7.6$ $\mathrm{Hz}, 1 \mathrm{H}, \mathrm{BZM}-\mathrm{H}_{4}$ ); IR (KBr) v: 3066, 2938, 2840, 1608 (S), 1579, 1497, 1461, 1259, $1026 \mathrm{~cm}^{-1}$. Anal. calcd for $\mathrm{C}_{32} \mathrm{H}_{26} \mathrm{ClN}_{5} \mathrm{O}_{3}$ : C 68.14, H 4.65, N 12.42; found $\mathrm{C}, 67.97$ $\mathrm{H} 4.51, \mathrm{~N} 12.65$.

3-[2-(4-氯苯氧甲基)苯并咪唑-1-亚甲基]-5,6-二(4甲氧基苯基)-1,2,4-三嗪(61): 苂光黄色晶体, 收率 $69 \%$. m.p. $176.5 \sim 177.9{ }^{\circ} \mathrm{C} ;{ }^{1} \mathrm{H}$ NMR (400 MHz, DMSO- $d_{6}$ ) $\delta$ : 3.75 (s, $\left.3 \mathrm{H}, \mathrm{CH}_{3} \mathrm{O}\right), 3.79$ (s, $\left.3 \mathrm{H}, \mathrm{CH}_{3} \mathrm{O}\right), 5.53$ (s, $2 \mathrm{H}$, $\left.\mathrm{CH}_{2} \mathrm{O}\right), 6.05\left(\mathrm{~s}, 2 \mathrm{H}, \mathrm{NCH}_{2}\right), 6.82 \sim 6.88(\mathrm{~m}, 4 \mathrm{H}, \mathrm{Ar}-\mathrm{H} \times 2$, Ar'-H×2), 6.99 (d, $J=8.8 \mathrm{~Hz}, 2 \mathrm{H}, \mathrm{Ar}^{\prime}-\mathrm{H}$ ), 7.20 (d, $J=9.2$ $\mathrm{Hz}, 2 \mathrm{H}, \mathrm{Ar}-\mathrm{H}$ ), 7.23 (d, $J=8.8 \mathrm{~Hz}, 2 \mathrm{H}, \mathrm{Ar}-\mathrm{H}$ ), $7.16 \sim 7.32$ $\left(\mathrm{m}, 2 \mathrm{H}, \mathrm{BZM}-\mathrm{H}_{5}, \mathrm{H}_{6}\right), 7.38\left(\mathrm{~d}, J=9.2 \mathrm{~Hz}, 2 \mathrm{H}, \mathrm{Ar}^{\prime}-\mathrm{H}\right)$, $7.67 \sim 7.73$ (m, 2H, BZM- $\left.\mathrm{H}_{4}, \mathrm{H}_{7}\right)$; IR (KBr) v: 3059, 2935, 2840, 1608 (S), 1579, 1494, 1461, 1259, $1030 \mathrm{~cm}^{-1}$. Anal. calcd for $\mathrm{C}_{32} \mathrm{H}_{26} \mathrm{ClN}_{5} \mathrm{O}_{3}$ : C 68.14, H 4.65, N 12.42; found C 68.39, H 4.87, N 12.60 .

3-[2-(4-硝基苯氧甲基)苯并咪唑-1-亚甲基]-5,6-二 (4-甲氧基苯基)-1,2,4-三嗪(6m): 黄色晶体, 收率 $68 \%$. m.p. $189.5 \sim 190.3{ }^{\circ} \mathrm{C} ;{ }^{1} \mathrm{H}$ NMR (400 MHz, DMSO- $d_{6}$ ) $\delta$ : 3.73 (s, $3 \mathrm{H}, \mathrm{CH}_{3} \mathrm{O}$ ), 3.79 (s, $\left.3 \mathrm{H}, \mathrm{CH}_{3} \mathrm{O}\right), 5.73$ (s, $2 \mathrm{H}$, $\left.\mathrm{CH}_{2} \mathrm{O}\right), 6.08\left(\mathrm{~s}, 2 \mathrm{H}, \mathrm{NCH}_{2}\right), 6.80(\mathrm{~d}, J=8.8 \mathrm{~Hz}, 2 \mathrm{H}$, Ar-H), 6.98 (d, $\left.J=8.8 \mathrm{~Hz}, 2 \mathrm{H}, \mathrm{Ar}^{\prime}-\mathrm{H}\right), 7.01$ (d, $J=9.2 \mathrm{~Hz}$, $\left.2 \mathrm{H}, \mathrm{Ar}^{\prime}-\mathrm{H}\right), 7.20$ (d, $\left.J=8.8 \mathrm{~Hz}, 2 \mathrm{H}, \mathrm{Ar}^{\prime}-\mathrm{H}\right), 7.23 \sim 7.34(\mathrm{~m}$, 2H, BZM-H $\left.\mathrm{H}_{5}, \mathrm{H}_{6}\right), 7.39$ (d, $\left.J=8.8 \mathrm{~Hz}, 2 \mathrm{H}, \mathrm{Ar}-\mathrm{H}\right), 7.66 \sim$ 7.77 (m, 2H, BZM-H $\left.\mathrm{H}_{4}, \mathrm{H}_{7}\right) 8.02$ (d, $\left.J=9.2 \mathrm{~Hz}, 2 \mathrm{H}, \mathrm{Ar}-\mathrm{H}\right)$; IR (KBr) v: 3082, 2938, 2840, 1608 (S), 1592, 1517, 1494, $1461,1344,1255,1017 \mathrm{~cm}^{-1}$. Anal. calcd for $\mathrm{C}_{32} \mathrm{H}_{26} \mathrm{~N}_{6} \mathrm{O}_{3}$ : C 66.89, H 4.56, N 14.63; found C 67.09, H 4.72, N 14.50.

3-[2-(1-菜氧甲基)苯并咪唑-1-亚甲基]-5,6-二(4-甲 氧基苯基)-1,2,4-三嗪(6n): 黄色晶体, 收率 70\%. m.p. $167.8 \sim 169.1{ }^{\circ} \mathrm{C} ;{ }^{1} \mathrm{H}$ NMR (400 MHz, DMSO- $d_{6}$ ) $\delta: 3.73$ (s, $\left.3 \mathrm{H}, \mathrm{CH}_{3} \mathrm{O}\right), 3.78\left(\mathrm{~s}, 3 \mathrm{H}, \mathrm{CH}_{3} \mathrm{O}\right), 5.74\left(\mathrm{~s}, 2 \mathrm{H}, \mathrm{CH}_{2} \mathrm{O}\right)$, $6.13\left(\mathrm{~s}, 2 \mathrm{H}, \mathrm{NCH}_{2}\right), 6.77$ (d, $\left.J=8.8 \mathrm{~Hz}, 2 \mathrm{H}, \mathrm{Ar}-\mathrm{H}\right), 6.93$ (d, $\left.J=8.8 \mathrm{~Hz}, 2 \mathrm{H}, \mathrm{Ar}^{\prime}-\mathrm{H}\right), 7.05$ (d, $J=8.8 \mathrm{~Hz}, 2 \mathrm{H}, \mathrm{Ar}^{\prime}-\mathrm{H}$ ), $7.12\left(\mathrm{~d}, J=8.4 \mathrm{~Hz}, 2 \mathrm{H}, \mathrm{Ar}^{\prime}-\mathrm{H}\right), 7.10 \sim 7.18(\mathrm{~m}, 1 \mathrm{H}, \mathrm{Ar}-\mathrm{H})$, $7.22 \sim 7.32\left(\mathrm{~m}, 3 \mathrm{H}, \mathrm{BZM}-\mathrm{H}_{5}, \mathrm{H}_{6}, \mathrm{Ar}-\mathrm{H}\right), 7.32 \sim 7.42(\mathrm{~m}$, $3 \mathrm{H}, \mathrm{Ar}-\mathrm{H}, \mathrm{Ar}-\mathrm{H} \times 2), 7.49(\mathrm{~d}, J=8.0 \mathrm{~Hz}, 1 \mathrm{H}, \mathrm{Ar}-\mathrm{H})$, $7.64 \sim 7.70(\mathrm{~m}, 1 \mathrm{H}, \operatorname{Ar}-\mathrm{H}), 7.72 \sim 7.78(\mathrm{~m}, 2 \mathrm{H}, \mathrm{BZM}-$ $\mathrm{H}_{4}, \mathrm{H}_{7}$ ); IR (KBr) v: 3056, 2935, 2840, $1608(\mathrm{~S}), 1579$, 1494, 1461, 1259, $1020 \mathrm{~cm}^{-1}$. Anal. calcd for $\mathrm{C}_{36} \mathrm{H}_{29} \mathrm{~N}_{5} \mathrm{O}_{3}$ : C 74.59, H 5.04, N 12.08; found C 74.42, H 4.91, N 12.19.

3-[2-(2-䒬氧甲基)苯并咪唑-1-亚甲基]-5,6-二(4-甲 氧基苯基)-1,2,4-三嗪(6o): 黄色晶体, 收率 72\%. m.p.
$141.0 \sim 142.3{ }^{\circ} \mathrm{C} ;{ }^{1} \mathrm{H}$ NMR (400 MHz, DMSO- $\left.d_{6}\right) \delta: 3.71$ (s, $\left.3 \mathrm{H}, \mathrm{CH}_{3} \mathrm{O}\right), 3.79\left(\mathrm{~s}, 3 \mathrm{H}, \mathrm{CH}_{3} \mathrm{O}\right), 5.64\left(\mathrm{~s}, 2 \mathrm{H}, \mathrm{CH}_{2} \mathrm{O}\right)$, 6.09 (s, 2H, NCH$)_{2}, 6.74$ (d, J=8.8 Hz, 2H, Ar-H), 6.83 (dd, $J=8.8,2.4 \mathrm{~Hz}, 1 \mathrm{H}$, Ar-H), 6.98 (d, $J=8.4 \mathrm{~Hz}, 2 \mathrm{H}$, Ar'-H), 7.17 (d, $J=8.8$ Hz, 2H, Ar'-H), $7.22 \sim 7.34$ (m, 2H, BZM- $\left.\mathrm{H}_{5}, \mathrm{H}_{6}\right), 7.35$ (d, $\left.J=8.8 \mathrm{~Hz}, 4 \mathrm{H}, \mathrm{Ar}^{\prime}-\mathrm{H}\right), 7.40 \sim 7.48$ (m, 1H, Ar-H), 7.65 7.75 (m, 4H, BZM- $\left.\mathrm{H}_{7}, \mathrm{Ar}-\mathrm{H} \times 3\right)$, $7.72\left(\mathrm{~d}, J=8.0 \mathrm{~Hz}, 1 \mathrm{H}, \mathrm{BZM}-\mathrm{H}_{4}\right)$; IR (KBr) $v: 3056,2944$, 2840, 1608 (S), 1503, 1461, 1255, $1026 \mathrm{~cm}^{-1}$. Anal. calcd for $\mathrm{C}_{36} \mathrm{H}_{29} \mathrm{~N}_{5} \mathrm{O}_{3}$ : C 74.59, H 5.04, N 12.08; found C 74.78, H 4.80, N 12.34 .

\subsection{3 目标化合物生物活性测试}

文献报道显示, $\mathrm{Cdc} 25 \mathrm{~B}$ 在人类许多癌症中是过度 表达的，寻找到高效特异的抑制剂对癌症研究与治疗可 提供新的工具和手段. PTP1B 是第一个被鉴定的蛋白酪 氨酸磷酸酯酶, 它通过对胰岛素受体的脱磷酰化, 进而 在调节胰岛素敏感性和脂肪代谢过程中起着非常重要 的作用. 因而，选择性的、高活性的 PTP1B 抑制剂在糖 尿病和肥胖症的治疗中有重要的价值.

目标化合物对 Cdc25B 和 PTP1B 的抑制活性篮选 实验按照参考文献 $[26,27]$ 的方法进行.

\subsubsection{Cdc25B 抑制实验}

应用大肠杆菌表达系统表达细胞分裂周期磷酸酯 酶(Cdc25B)催化结构域, 以谷胱甘肽 S-转移酶(GST)融 合蛋白形式存在于上清, 细胞裂解后经 GSH-亲和柱纯 化, 获得活性 GST 融合的 Cdc25B 蛋白. 采用苂光底 物邻甲基荧光磷酸酯(OMFP), 观察不同化合物对重组 酶的活性的抑制. OMFP 水解产物邻甲基苂光素(OMF) 在被 $485 \mathrm{~nm}$ 激发光激发后可发射出波长为 $535 \mathrm{~nm}$ 的可 检测的荧光信号，从而观察酶的活性变化以及化合物对 其的抑制情况. 实验中采用的阳性参照化合物为 $\mathrm{Na}_{3} \mathrm{VO}_{4}$.

\subsubsection{PTP1B 抑制实验}

用于篮选的蛋白酪氨酸磷酸酯酶(PTP1B) 是从大肠 杆菌中表达并纯化的 GST 融合蛋白. 采用紫外底物对 硝基苯磷酸二钠 $(p \mathrm{NPP})$, 观察不同化合物对重组酶的活 性抑制, 以初步评价化合物的药用效果. PTP1B 水解底 物 $p$ NPP 的磷酯得到的产物在 $405 \mathrm{~nm}$ 处有很强的光吸 收. 因此可以直接监测 $405 \mathrm{~nm}$ 处光吸收的变化以观察 酶的活性变化以及化合物对其的抑制情况. 实验中采用 的对照化合物为齐墩果酸.

辅助材料(Supporting Information) 所有目标化合物 的 IR 和 ${ }^{1} \mathrm{H}$ NMR 谱图. 这些材料可以免费从本刊网站 (http://sioc-journal.cn/)上下载. 


\section{References}

[1] Stýskala, J.; Stýskalová, L.; Slouka, J.; Hajdúch M. Eur. J. Med. Chem. 2008, 43, 449.

[2] Nassar, I. F. J. Heterocycl. Chem. 2013, 50, 129.

[3] Al-Adiwish, W. M.; Tahir, M. I. M.; Siti-Noor-Adnalizawati, A.; Hashim, S. F.; Ibrahim, N.; Yaacob, W. A. Eur. J. Med. Chem. 2013, 64, 464

[4] Mashaly, M. M.; El-Shafiy, H. F.; El-Maraghy, S. B.; Habib, H. A. Spectrochim. Acta, Part A 2005, 61, 1853.

[5] Khoshneviszadeh, M.; Ghahremani, M. H.; Foroumadi, A.; Miri, R.; Firuzi, O.; Madadkar-Sobhani, A.; Edraki, N.; Parsa, M.; Shafiee, A. Bioorg. Med. Chem. 2013, 21, 6708.

[6] Zhan, P.; Li, X.; Li, Z.-Y.; Chen, X.-W.; Tian, Y.; Chen, W.-M.; Liu, X.-Y; Pannecouque, C.; Clercq, E. D. Bioorg. Med. Chem. Lett. 2012, 22, 7155.

[7] Ban, K.; Duffy, S.; Khakham, Y.; Avery, V. M.; Hughes, A.; Montagnat, O.; Katneni, K.; Ryan, E.; Baell, J. B. Bioorg. Med. Chem. Lett. 2010, 20, 6024.

[8] Li, Y.-J.; Shao, X.; Gao, L.-X.; Jin, K.; Sheng, L.; Luo, T.-C.; Yu, Y.; Li, J. Chin. J. Org. Chem. 2013, 33, 2178 (in Chinese). (李英俊, 邵昕, 高立信, 靳焜, 盛丽, 罗潼川, 于洋, 李佳, 有 机化学, 2013, 33, 2178.)

[9] Li, Y. J.; An, J.; Jin, K.; Sun, S.-Q.; Lv, C.-W.; Chen, Q.; Chen, X.-Y. Chem. Reag. 2012, 34, 497 (in Chinese). (李英俊, 安静, 靳焜, 孙淑琴, 吕成伟, 陈晴, 陈晓雨, 化学试 剂, 2012, 34, 497.)

[10] Marandi, F.; Jangholi, M.; Hakimi, M.; Rudbari, H. A.; Bruno, G. J. Mol. Struct. 2013, 1063, 71.

[11] Irannejad, H.; Kebriaieezadeh, A.; Zarghi, A.; Montazer-Sadegh, F.; Shafiee, A.; Assadieskandar, A.; Amini, M. Bioorg. Med. Chem. 2014, 22, 865 .

[12] Irannejad, H.; Amini, M.; Khodagholi, F.; Ansari, N.; Tusi, S. K.; Sharifzadeh, M.; Shafiee, A. Bioorg. Med. Chem. 2010, 18, 4224.

[13] Khodaghol, F.; Tusi, S. K.; Alamdary, S. Z.; Amini, M.; Ansari, N.
Toxicol. In Vitro 2012, 26, 907.

[14] Ali, T. E. S. Eur. J. Med. Chem. 2009, 44, 4385.

[15] Thirumurugan, P.; Perumal, P. T. Dyes Pigm. 2011, 88, 403

[16] Panayiotidou, L.; Stylianou, M.; Arabatzis, N.; Drouza, C.; Lianos, P.; Stathatos, E.; Keramidas, A. D. Polyhedron 2013, 52, 856.

[17] Husain, A.; Rashid, M.; Shaharyar, M.; Siddiqui, A. A.; Mishra, R. Eur. J. Med. Chem. 2013, 62, 785.

[18] Wang, X.-J.; Chu, N.-Y.; Wang, Q.-H.; Liu, C.; Jiang, C.-G.; Wang, X.-Y.; Ikejima, T.; Cheng, M.-S. Bioorg. Med. Chem. Lett. 2012, $22,6297$.

[19] Song, W.-J.; Cheng, J.-P.; Jiang, D.-H.; Guo, L.; Cai, M.-F.; Yang, H.-B.; Lin, Q.-Y. Spectrochim. Acta, Part A 2014, 121, 70.

[20] Desai, N. C.; Shihory, N. R.; Kotadiya, G. M. Chin. Chem. Lett. 2014, 25, 305.

[21] Gaba, M.; Singh, S.; Mohan, C. Eur. J. Med. Chem. 2014, 76, 494.

[22] Tremblay, M.; Bonneau, P.; Bousquet, Y.; DeRoy, P.; Duan, J.; Duplessis, M.; Gagnon, A.; Garneau, M.; Goudreau, N.; Guse, I.; Hucke, O.; Kawai, S. H.; Lemke, C. T.; Mason, S. W.; Simoneau, B.; Surprenant, S.; Titolo, S.; Yoakim, C. Bioorg. Med. Chem. Lett. 2012, 22, 7512.

[23] Lee, K.; Goo, J.-I.; Jung, H. Y.; Kim, M.; Boovanahalli, S. K.; Park, H. R.; Kim, M.-O.; Kim, D.-H.; Lee, H. S.; Choi, Y. Bioorg. Med. Chem. Lett. 2012, 22, 7456.

[24] Li, Y.-J.; Liu, L.-J.; Jin, K.; Sun, S.-Q.; Xu, Y.-T. Acta Chim. Sinica 2010, 68, 1577 (in Chinese).

(李英俊, 刘丽军, 靳焜, 孙淑琴, 许永廷, 化学学报, 2010, 68, 1577.)

[25] Sun, Y.-F.; Pan, W.-L.; Wu, R.-T.; Song, H.-C. Chin. J. Org. Chem. 2006, 26, 1079 (in Chinese). (孙一峰，潘文龙，吴仁涛，宋化灿，有机化学, 2006, 26, 1079.)

[26] Huang, W.-G.; Jiang, Y.-Y.; Li, Q.; Li, J.; Li, J.-Y.; Lu, W.; Cai, J.-C. Tetrahedron 2005, 61, 1863.

[27] Sun, L.-P.; Shen, Q.; Piao, H.-H.; Ma, W.-P.; Gao, L.-X.; Zhang, W.; Nan, F.-J.; Li, J.; Piao, H.-R. Eur. J. Med. Chem. 2011, 46, 3630 .

(Li, L.; Fan, Y.) 